\title{
Vagus nerve stimulation recruits the central cholinergic system to enhance perceptual learning
}

Kathleen A. Martin ${ }^{1-5}$, Eleni S. Papadoyannis ${ }^{6}$, Jennifer K. Schiavo ${ }^{1-4}$, Saba Shokat Fadaei $^{1-4}$, Nesibe Z. Temiz ${ }^{7}$, Matthew J. McGinley ${ }^{8-9}$, David A. McCormick ${ }^{10}$, Robert C. Froemke ${ }^{1-5,{ }^{*}}$

1 Skirball Institute for Biomolecular Medicine, New York University School of Medicine, New York, NY, USA

${ }^{2}$ Neuroscience Institute, New York University School of Medicine, New York, NY, USA

${ }^{3}$ Department of Otolaryngology, New York University School of Medicine, New York, NY, USA

${ }^{4}$ Department of Neuroscience and Physiology, New York University School of Medicine, New York, NY, USA

${ }^{5}$ Center for Neural Science, New York University, New York, NY, USA

${ }^{6}$ Princeton Neuroscience Institute, Princeton University, Princeton, New Jersey

7 Friedrich Miescher Institute for Biomedical Research, Basel, Switzerland

8 Duncan Neurological Research Institute, Baylor College of Medicine, Houston TX 77030

9 Department of Neuroscience, Baylor College of Medicine, Houston TX 77030

10 Institute of Neuroscience, University of Oregon, Eugene OR 97403

* correspondence to: robert.froemke@med.nyu.edu 


\begin{abstract}
Perception can be refined by experience up to certain limits. It is unclear if limitations are absolute or could be partially overcome via enhanced neuromodulation and/or plasticity. Recent studies highlight the utility of peripheral nerve stimulation - specifically vagus nerve stimulation (VNS) - for altering neural activity and augmenting experiencedependent plasticity, although little is known about central mechanisms recruited by VNS. Here we developed an auditory discrimination task for mice implanted with a VNS electrode. VNS occurring during behavior gradually improved discrimination abilities beyond the level achieved by training alone. Using two-photon imaging, we identified changes to auditory cortical responses and activation of cortically-projecting cholinergic axons with VNS. Anatomical and optogenetic experiments revealed that VNS seemed to enhance task performance via activation of the central cholinergic system. These results highlight the importance of cholinergic modulation for the efficacy of VNS, perhaps enabling further refinement of VNS methodology for clinical conditions.
\end{abstract}

\title{
Introduction
}

Sensory processing is refined over development and must continue to be adaptive throughout life, in order to adequately regulate behavior in dynamic and challenging environments. This requires that adult perceptual and cognitive abilities are not fixed, but rather can be improved with additional experience and training. Perceptual learning, i.e., improvement in sensory perceptual abilities with practice, has been shown to occur across a wide range of domains, including visual processing of orientation, binocularity, and other spatial features ${ }^{1-7}$, auditory processing of pitch and temporal intervals ${ }^{8-12}$, and the detection and recognition of sensory stimuli in other modalities ${ }^{13-15}$. However, changes in perceptual abilities can often be quite limited and stimulus-specific, even after extensive periods of training ${ }^{5,16-18}$. It is generally believed that adult perceptual learning across species relies on neural mechanisms of synaptic plasticity within the cerebral cortex ${ }^{1,7,14,17}$, but it remains unclear what factors might limit cortical plasticity achieved by perceptual training and any consequent changes in perception and behavior. Conversely, mechanisms for plasticity not engaged during training might then be available for recruitment by other means, and perhaps improve sensory perceptual abilities even further.

Studies of the mammalian auditory system have proved to be especially revealing for determining the mechanisms connecting perceptual learning and neural plasticity. A long literature has connected changes in auditory experience with long-lasting neural changes in the central auditory system. In particular, many studies have demonstrated how auditory training affects tonotopic maps and single neuron responses, generally showing that acoustic stimuli predictive of outcome have enhanced representations in the auditory 
cortex $8,9,11,12,19,20$. Despite clear evidence that behavioral training eventually affects cortical maps and receptive fields, it is less clear how these changes are initially induced and occur throughout experience and learning to produce perceptual changes ${ }^{21,22}$. One consistent finding across systems and species, however, is that central neuromodulatory systems such as the cholinergic basal forebrain are important for sensory perceptual learning and cortical plasticity ${ }^{23-36}$. However, it remains unclear the extent to which modulatory systems are endogenously recruited under different contexts or forms of training, and if it might be possible to artificially leverage central modulation to further improve perceptual learning.

Peripheral nerve stimulation provides an opportunity to augment performance and lead to lasting improvements in auditory perceptual abilities via activation of neuromodulatory systems. Vagus nerve stimulation (VNS) has been used for several clinical applications in humans, including treating epilepsy ${ }^{37,38}$, alleviating treatment-resistant depression ${ }^{39}$, and motor deficits after stroke ${ }^{40}$. Despite its well-established benefits, less is known about the circuit mechanism through which VNS acts. This has led to experimental approaches for studying VNS in a range of species including rats ${ }^{41,42}$, ferrets ${ }^{43}$, and non-human primates ${ }^{44}$. Recently, in collaboration we developed and validated a VNS cuff electrode for mice ${ }^{45-48}$ to investigate the role of neuromodulation in VNS-mediated motor and perceptual learning. Previous work in non-human model organisms has shown that vagus nerve stimulation can induce neuroplasticity in primary sensory areas ${ }^{41,42,49}$ and in motor areas $^{46,50,51}$. This enhanced neuroplasticity is thought to be partially mediated by indirectly activating neuromodulatory networks, including noradrenergic neurons in locus coeruleus $^{48,52,53}$ and cholinergic neurons in basal forebrain ${ }^{46-48}$ via the nucleus tractus solitarii (NTS) ${ }^{54,55}$. With the development of VNS cuffs for mice, we aimed to study the circuit mechanisms and modulatory systems activated by VNS, asking how VNS might be applied to promote plasticity and improve auditory perceptual learning.

\section{Results}

\section{Auditory perceptual training in head-fixed mice}

We first developed an auditory task to parametrically quantify the psychophysical abilities of mice for frequency discrimination. A total of 29 mice ( 5 female wild-type, 11 male wildtype, 5 female ChAT-Cre, 8 male ChAT-Cre) were used for behavioral studies; animals were head-fixed and progressively trained to classify presented pure tones in a twoalternative forced-choice (2AFC) task for a water reward. On each trial, mice were presented with an auditory stimulus of one frequency ranging between 4-38 kHz at $70 \mathrm{~dB}$ sound pressure level (SPL). Mice were trained to lick left in response to tones of one specific 'center' frequency (varied between 11-16 kHz across individual animals), and to lick right for tones of any other 'non-center' frequency (Fig. 1a). There were eight non- 
center tones with four tones up to 1.5 octaves lower than the center frequency, and four tones up to 1.5 octaves higher than the center frequency in the full training set (Fig. 1b).

Mice learned the task in three stages, with additional frequencies added as individual performance improved. During stage 1 (first shaping stage), we only presented two frequencies (the 'center' tone and one 'non-center' tone 1.5 octaves from center; Fig. 1b, light blue). Animals completed stage 1 after reaching our performance criterion ( $\geq 80 \%$ correct) for three consecutive days (Fig. 1c). The number of days to reach performance criteria in stage 1 was variable across individual mice, from 5-37 days (Fig. 1d,e). There was no significant difference in performance across stage one by sex or genotype (wildtype vs ChAT-Cre in C57BI/6J background; wild-type males: $10.6 \pm 3.4$ days in stage 1, mean \pm s.d.; wild-type females: $16.4 \pm 7.7$ days; ChAT-Cre males: $12.4 \pm 3.1$ days; ChATCre females: $8.8 \pm 6.4$ days; $p=0.44$ for genotype, $p=0.52$ for sex, two-way ANOVA across all four groups with Tukey's multiple comparisons correction). Following stage 1, we then introduced one other 'non-center' frequency that was 1.5 octaves away from the center frequency in the opposite direction (Fig. 1b, medium blue), and trained animals in this second shaping stage 2 for three days (Fig. 1c,e). We then introduced all other frequencies (stage 3; Fig. 1c,e, N=29 mice), with all animals being trained in stage 3 for at least nine days. Performance improved across non-center frequencies throughout stage 3 (Fig. 1f, $\mathrm{N}=29$ mice, day 1 of stage 3 compared to maximum performance, minimum of 3 days of stable performance).

Mice correctly identified stimuli as center or non-center tones at variable rates across animals (Fig. 1f). On the day of peak performance, animals correctly identified frequencies as the center frequency at $79.6 \pm 12.0 \%$ of the time $(\mathrm{N}=29$ animals, mean \pm s.d., minimum 3 days of stable performance). Animals generally had stable performance across days after at least nine days in the full version of the task (Fig. 1g). Even after performance stabilized, animals continued to make a significant number of errors on the non-center frequencies closest to the center, either a quarter octave or a half octave away from the center frequency (Fig. $1 \mathbf{h}$; error rates at \pm 0.25 octaves: $61.7 \pm 25.3 \%$; error rates at \pm 0.5 octaves: $42.7 \pm 26.4 \%$, mean \pm s.d.; significantly higher errors compared to center tone responses, $\mathrm{p}<0.001)$. These data show that while head-fixed mice have relatively stable frequency discrimination abilities, their performance is still sub-optimal even after days to weeks of continued positive reinforcement to correctly resolve frequencies within

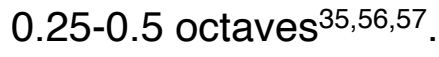

\section{Longitudinal vagus nerve stimulation in mice}

Our behavioral results showed that, once a certain level of performance was obtained, additional training did not consistently refine perceptual abilities in an individual mouse 
2 bioRxiv preprint doi: https://doi.org/10.1101/2022.01.28.478197; this version posted January 28, (which was not certified by peer review) is the author/funder. All rights reserved. No reuse

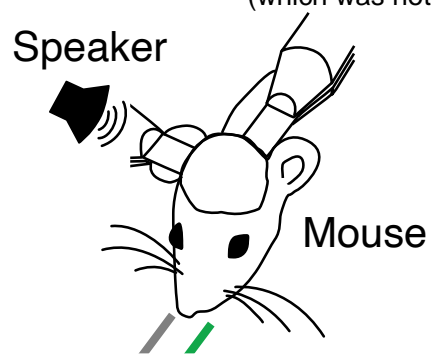

Lick ports

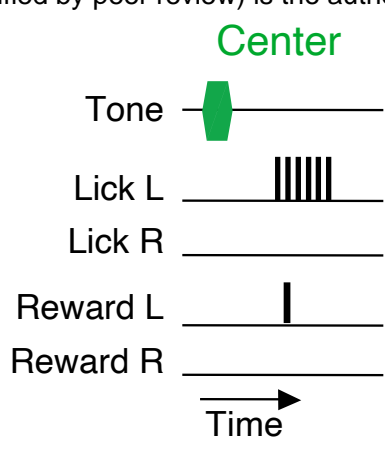

ITI

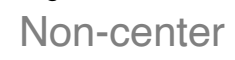

C

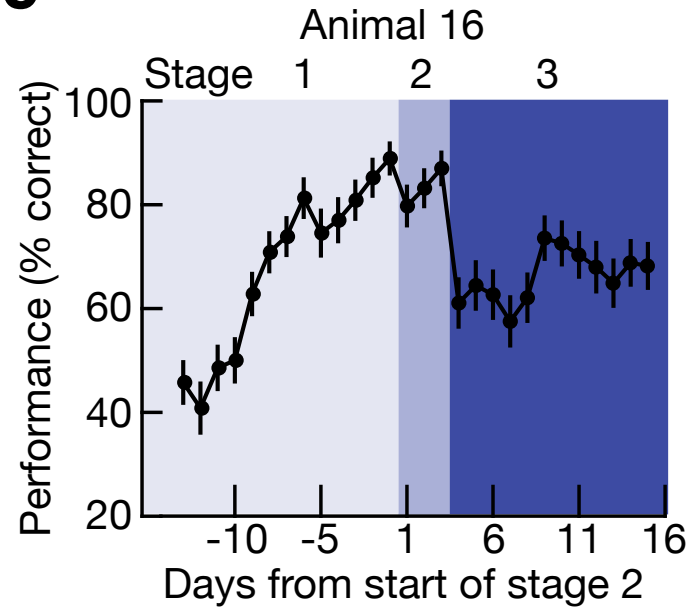

0

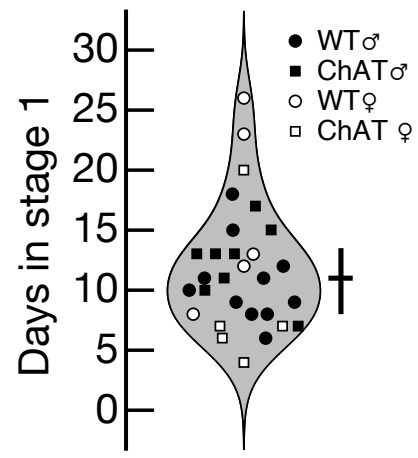

9

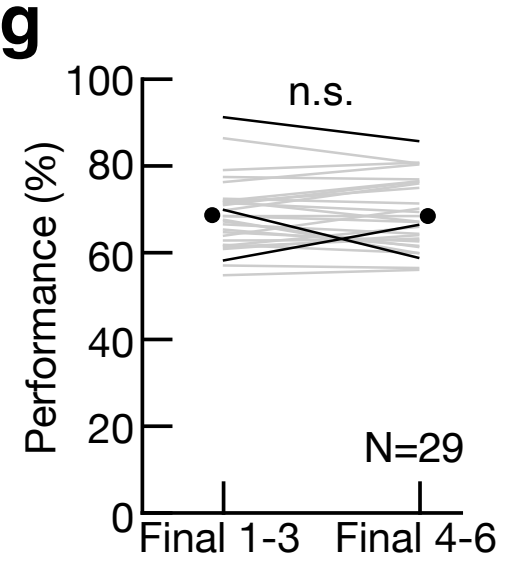

Days of stable performance

e

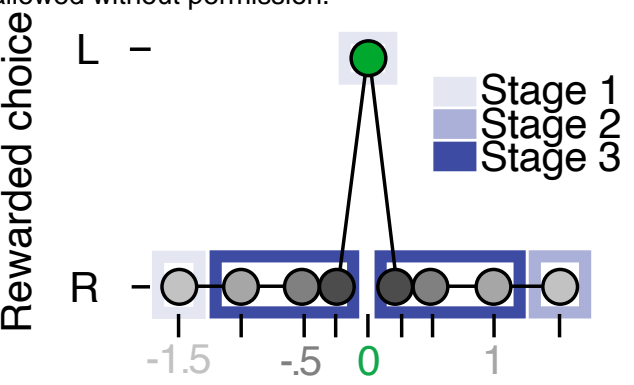

Dist from center (octaves)

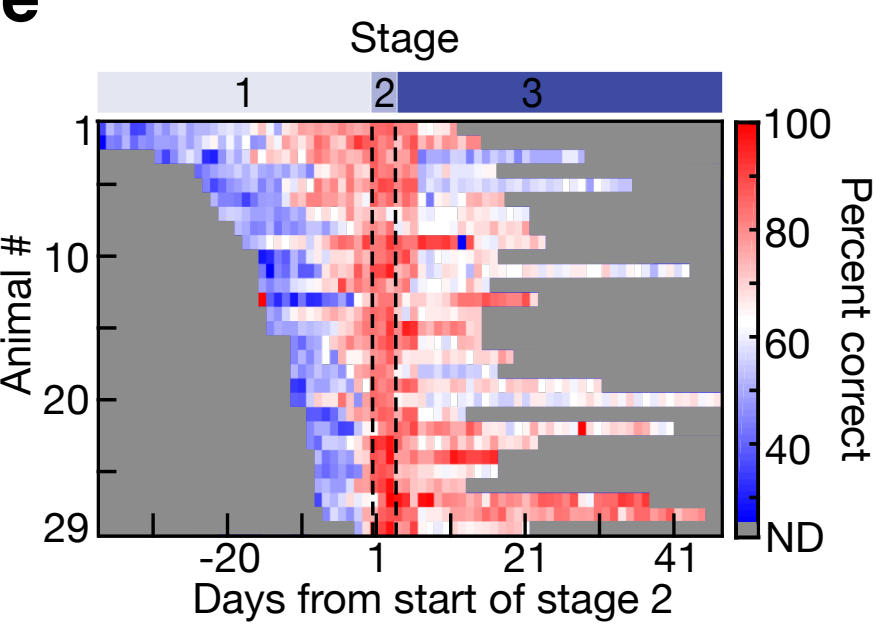

h
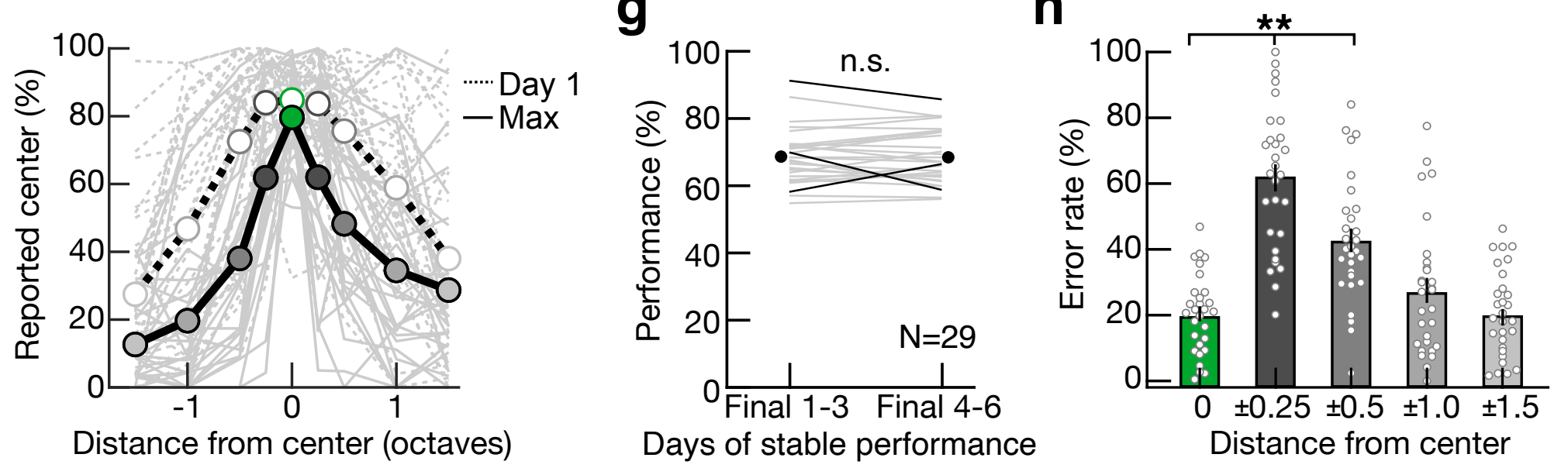

Figure 1 
Figure 1. 2AFC task for mouse auditory frequency discrimination. a, Behavioral schematic showing trial structure. On each trial, a head-restrained and water-restricted mouse is presented with a pure tone of a single frequency for 0.25 seconds at $70 \mathrm{~dB}$ SPL. Mice were trained to classify tones as either the center frequency (green) or non-center (shades of gray, with dark gray indicating closest to the center tone frequency and light gray indicating furthest from the center) by licking left (for center) or right (for non-center) in a response epoch $0.25-2.5$ seconds from tone presentation. If tones were classified correctly, reward was delivered on the corresponding lick port. Inter-trial interval (ITI), 2.5 to 7 seconds. b. Set of auditory training stimuli. The center frequency $(11.3-16.0 \mathrm{kHz}$, specified per animal) was rewarded if the animal licked left; non-center frequency $( \pm 0.25$ 1.5 octaves from center frequency) was rewarded if the animal licked right. A single noncenter frequency either -1.5 or 1.5 octaves from center tone was used in stage 1 , the other \pm 1.5 octave frequency was added in stage 2 , all other stimuli added in stage 3 . c, Mean performance across all three stages for example wild-type male mouse. When performance reached $\geq 80 \%$ for three consecutive days in stage 1 (lightest blue), this animal was transitioned to stage 2 (middle blue) for three days before moving to stage 3 . Error bars, binomial confidence interval. d, Number of days in stage 1 for all animals (median 11 days, inter-quartile range 8-15 days. Open circles, wild-type females $(\mathrm{N}=5)$; filled circles, wild-type males ( $N=11)$; open squares, ChAT-Cre females $(N=5)$; filled squares, ChAT-Cre males $(\mathrm{N}=8)$. e, Performance for all animals relative to day 1 of stage $2(\mathrm{~N}=29$ mice). Heat map, \% correct performance for each stage of training. Gray, no data (ND) as animals were not trained on those days. f, Percent reported center (i.e., licked left) across all stimuli in on day one of stage 3 (dotted lines) and day of maximum performance (solid lines). Gray lines, individual mice. Colored circles, means ( $N=29)$. g, Stability of individual performance at end of stage 3 (before starting VNS). Mean overall performance was stable for final six days of stage three; final days 1-3 (performance: $68.7 \pm 1.5 \%$ correct, $\mathrm{N}=29$, mean \pm s.e.m) compared to final days 4-6 (performance: $68.4 \pm 1.5 \%, p=0.76$ compared to final days $4-6$, Student's two-tailed paired t-test). Individually, only $3 / 29$ animals showed a significant difference $(p<0.05$, Student's twotailed paired t-test) on final days 1-3 vs final days 4-6 (bold lines), 26/29 animals had individually stable performance (thin lines). n.s., not significant. $\mathbf{h}$, Error rate for each stimulus. Small circles, individual mice. Error rate was significantly higher at \pm 0.25 and 0.5 octaves (dark gray, \pm 0.25 octave error rate: $61.7 \pm 4.1 \%$, mean \pm s.e.m; medium gray, \pm 0.5 octave error rate: $42.7 \pm 3.6 \%$ ) compared to center (green, center tone error rate; $20.4 \pm 2.3 \%, p<0.001$ compared to $\pm 0.25-0.5$, one-way ANOVA with Tukey's multiple comparisons correction). ${ }^{* *}, p<0.001$. 
beyond a certain level. This is consistent with a long literature on psychophysics and perceptual learning in humans and other species ${ }^{5,16-18,35}$. We wondered if this was a fixed perceptual limit set by physical limitations of the sensory epithelium or if it could be improved by engagement of central mechanisms of neuromodulation and plasticity. Methods such as transgene expression and optogenetic stimulation can lead to lasting behavioral gains in rodents ${ }^{21}$, but these approaches are currently infeasible or impractical in many species including human subjects. However, stimulation of the vagus nerve has recently been shown to influence neural responses in the central nervous system, altering physiological and cognitive variables $39,41,52,53,58$. Some of these effects may be due to indirect activation of central modulation, but little is known about the underlying mechanisms by which VNS initiates prolonged enhancement of behavior.

We adapted the bipolar VNS cuff used in rats ${ }^{41}$ to make custom vagus nerve cuff electrodes for use in mice ${ }^{45,47,48}$ and verified successful stimulation of the vagus nerve by measuring impedance of the cuff electrode. We implanted custom cuff electrodes around the left vagus nerve in adult mice (Fig. 2a). We measured cuff impedance daily in awake animals for days to months post-implantation over the course of behavioral training (Fig. 2b). Cuff impedances $<10 \mathrm{k} \Omega(0.03-5.41 \mathrm{k} \Omega)$ were considered viable for VNS, and many animals had stable cuff impedances in this range for weeks (Fig. 2c). At much higher cuff impedance values (e.g., $115 \mathrm{M} \Omega$; Extended Data Fig. 1), VNS was ineffective at affecting physiological variables such as breathing rate, peripheral blood oxygen level $\left(\mathrm{SpO}_{2}\right)$, or heart rate, consistent with our previous work ${ }^{45}$. Therefore, animals with impedance values over $10 \mathrm{k} \Omega$ were considered as 'sham' implanted.

\section{Vagus nerve stimulation improves auditory perceptual learning over days}

We next asked how VNS might affect the performance of fully trained animals on the 2AFC task. After several days of training on stage 3 (average 16.9+/-10.8 days, range 636 days), we implanted 11 trained mice with a vagus nerve cuff and monitored impedances daily. Animals then recovered for about a week with ad lib water, and training on the 2AFC task was reinstated after 4-10 days of additional water restriction (total days in stage 3: average 20.9 11 .0 days, range 9-40 days). VNS occurred during behavior in a blockwise manner, with a 'block' being a set of 50 trials. In blocks 1, 3, and 5 there was no VNS and training was identical to that previously described in Figure 1. In blocks 2 and 4, VNS was performed concurrently with tone presentation, essentially being paired with all center and non-center stimuli. Specifically, we stimulated the vagus nerve for 500 ms at $30 \mathrm{~Hz}$ with a $100 \mu$ s pulse width centered around the $250 \mathrm{~ms}$ tone, such that VNS began 125 ms before tone onset and continued for $125 \mathrm{~ms}$ after tone offset (Fig. 2d). By design, this meant that VNS pairing was performed irrespective of trial outcome, in contrast to a recent important study of motor learning (Bowles, et al., 2021). VNS 
stimulation intensity was 0.6-0.8 $\mathrm{mA}$, fixed per animal. Prior to the start of VNS pairing, implanted animals were trained for 3-7 days post-implantation without VNS, to establish a new baseline level of performance and ensure that this was comparable to their preimplantation behavior (Fig. 2e, $\mathrm{N}=11$ mice, change in performance: $0.6 \pm 1.4 \%$, meants.e.m.).

We found that VNS pairing led to enduring improvements in 2AFC task performance for most animals, which emerged gradually over days and largely enhanced correct responses to the \pm 0.25 and \pm 0.5 octave flanking non-center tones (Fig. 2f). We initially quantified the change in performance across all stimuli over all five training blocks (' $\Delta \%$ correct') as the overall correct rate on each day relative to the average performance on the final three days of training prior to cuff implantation. The maximum change in 2AFC task performance occurred several days after initiating VNS pairing during training (Fig. $\mathbf{2 f , g}$, best performance after $11.6 \pm 7.1$ days, $\mathrm{N}=11$ mice, mean \pm s.d.). We compared behavioral performance on the best day from VNS pairing $( \pm 1$ day, to include the day before and after the best day for each animal, averaging over those three days) to the baseline behavioral performance on the three days of training just before the start of VNS pairing. Behavioral performance significantly increased (Fig. $\mathbf{2 h}, \mathbf{N}=11$ mice, before VNS: 73.0 $\pm 2.4 \%$ correct over all center/non-center stimuli, best day after VNS: $80.2 \pm 1.8 \%$ correct over all stimuli, $\mathrm{p}=0.002$, Student's two-tailed paired t-test).

As VNS occurred with presentation of both center and non-center stimuli, we asked if performance at certain frequencies were specifically improved after days of VNS pairing. We noticed that, on average, behavioral performance was most improved at the noncenter tones flanking the center (Fig. 2i). We quantitatively compared behavioral performance at individual frequencies on the day with maximum performance after VNS pairing and baseline performance (Fig. 2i, $\mathrm{N}=11$ mice; all 11 animals individually displayed in Extended Data Fig. 2). Most of the improvement in behavioral performance came from reductions in errors at non-center frequencies $\pm 0.25,0.5$ and 1 octave from the center frequency (Fig. $\mathbf{2 j}$, change in error at \pm 0.25 octaves from center: $-15.1 \pm 8.0 \%$, $p=0.038$, Student's one-tailed paired t-test; change in error at \pm 0.5 octaves: $-13.4 \pm 7.0 \%$, $p=0.035$; change in error at \pm 1 octave: $-7.3 \pm 4.1 \%, p=0.045)$. In contrast, responses to the center stimulus were generally unaffected (Fig. 2i, before VNS: $80.0 \pm 5.0 \%$ correct at 0 , best days after VNS: $84.1 \pm 2.9 \%$ correct at $0, N=11, p=0.18$, Student's one-tailed paired t-test; Fig. 2j, change in error at 0: $-4.16 \pm 4.6 \%, p=0.18$, Student's one-tailed paired ttest). The increase in performance resulting from enhancements only at $\pm 0.25-0.5$ octaves of center (Fig. 2k, 'VNS', $p=0.03$, Student's one-tailed paired t-test) were not observed for \pm 0.25-0.5 octave stimuli in animals receiving sham VNS for 11-13 days with electrode impedances $>10 \mathrm{k} \Omega$ (Fig. 2k, 'Sham', $\mathrm{N}=6$ mice, $\mathrm{p}=0.33$ ), where this 11-13 day 

(which was not certified by peer reviev, Is the author/funder. All rights reserved. No reuse allowed witho
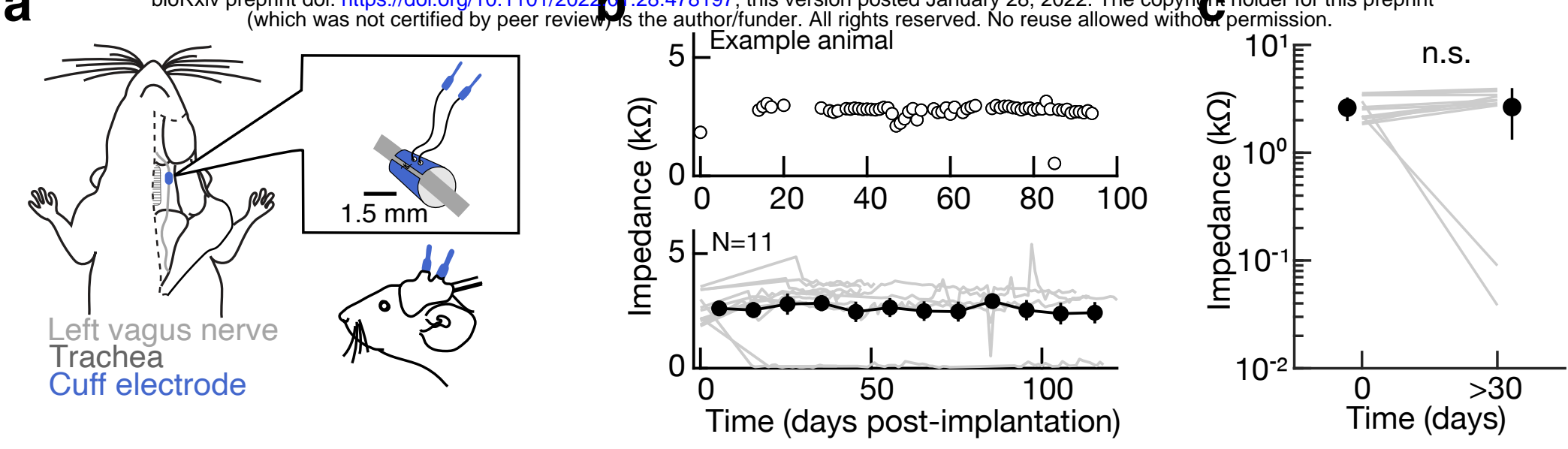

Speaker

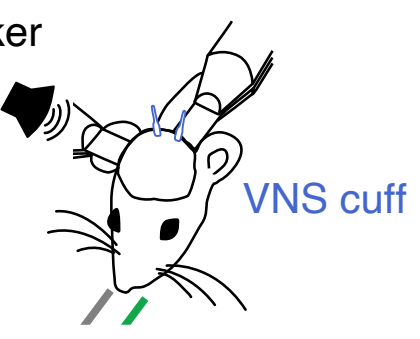

Lick ports e

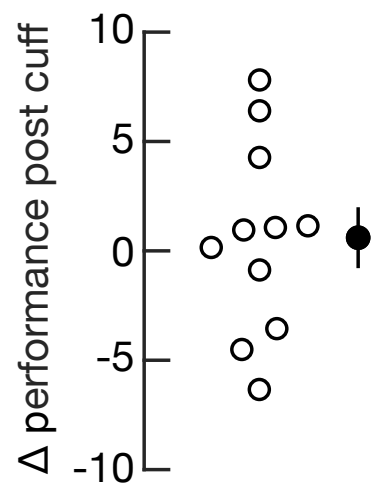

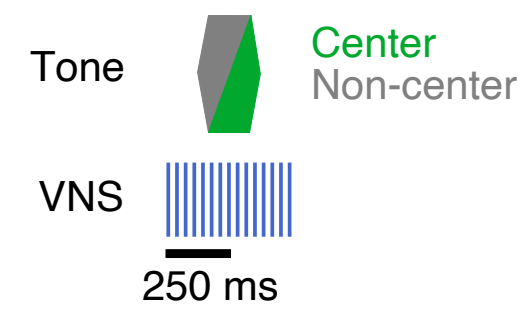

f

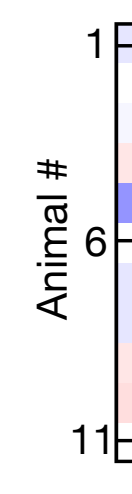

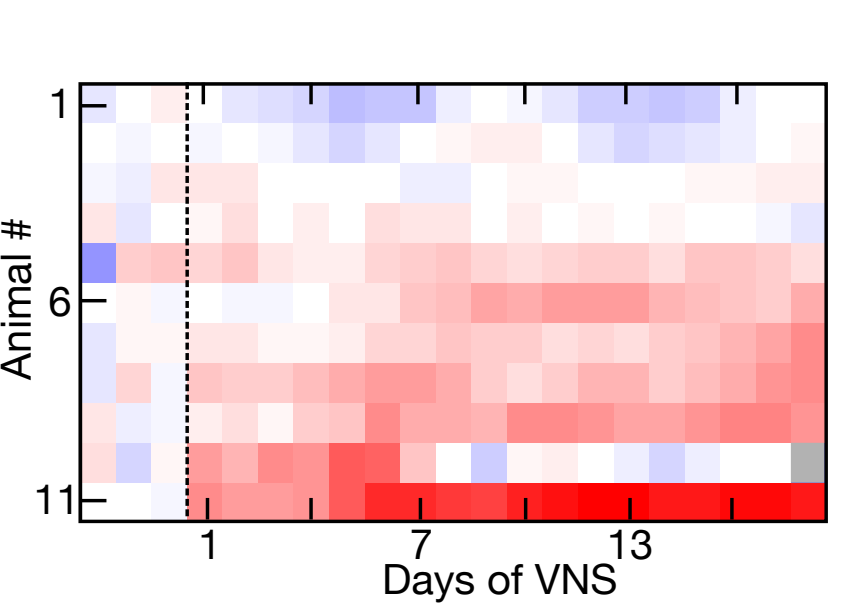

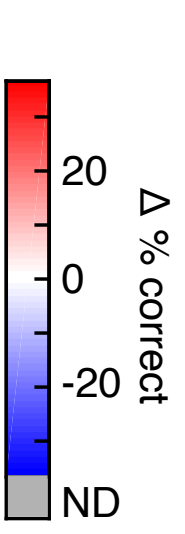

k
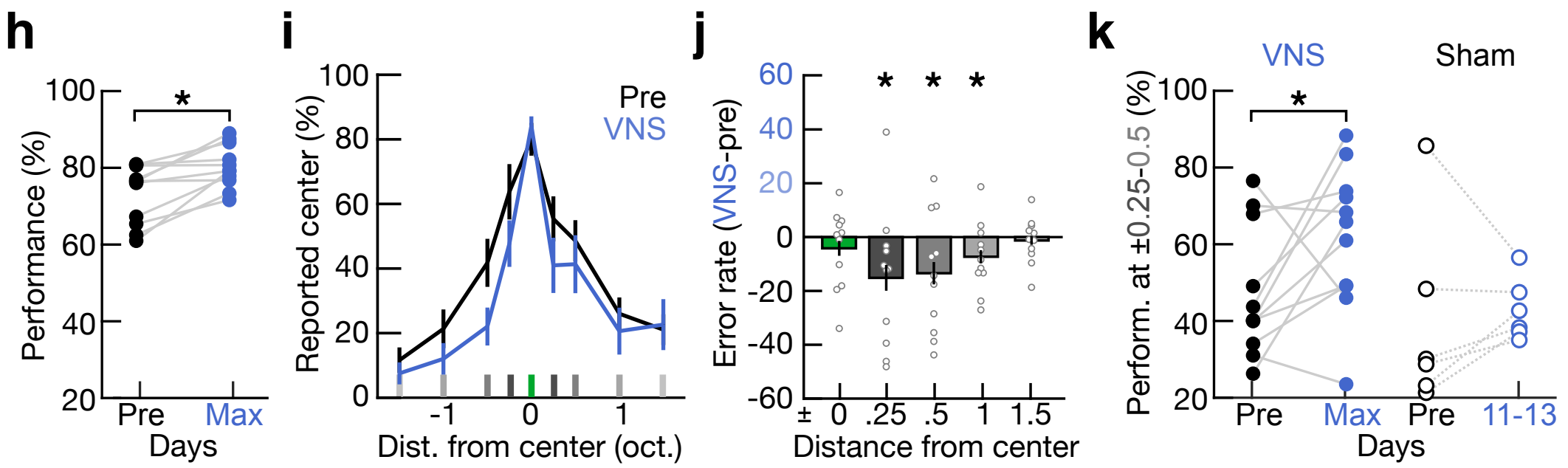

Figure 2 
Figure 2. VNS during behavior improves perceptual discrimination over days. a, Schematic of cuff electrode implantation on mouse left vagus nerve. b. Impedance over days post-implantation from a representative animal (top) and all 11 wild-type mice (bottom; 3 female, 8 male) used for VNS pairing behavioral experiments. Gray, individual animals; black, mean \pm s.d. every 10 days $(\mathrm{N}=11$ mice). c, Cuff impedance is stable over time. Impedance reading on day 0 (day of cuff implantation) and the first measurement $30+$ days after implantation (30-54 days) (Day 0 mean: $2.61 \pm 0.65 \mathrm{k} \Omega$, day $30-54$ mean: $2.65 \pm 1.33 \mathrm{k} \Omega$, mean \pm s.d., $N=11$ mice, $p=0.93$, Student's two-tailed paired t-test). $d$, Schematic of VNS pairing during behavior. VNS was performed in two blocks of 50 trials (blue, blocks 2 and 4), interleaved between blocks of no stimulation (gray, blocks 1, 3 and 5). VNS parameters: 500 ms duration, $30 \mathrm{~Hz}$ rate, 0.6-0.8 $\mathrm{mA}$ intensity, centered around the tone for that trial. During blocks of VNS, stimuli of all frequencies (center and non-center) were presented during behavior. e, Performance is not affected by VNS cuff implantation $(0.6 \pm 1.34 \%$, mean \pm s.e.m., $\mathrm{N}=11, \mathrm{p}=0.99$, Student's two-tailed paired t-test). $\mathbf{f}$, VNS pairing during behavior gradually improved performance over days ( $\mathrm{N}=11$ mice), $\mathbf{g}$, Days to maximum performance per animal after initiation of VNS pairing during behavior. $\mathbf{h}$, Performance over all stimuli improves after VNS pairing. Percent correct on day with maximum performance $( \pm 1$ day) of VNS pairing across all frequencies (blue, 'Max', $80.2 \pm 1.8 \%$, mean \pm s.e.m., $\mathrm{N}=11$ ) in comparison to the behavior three days prior to VNS (black, 'Pre', $73.0 \pm 2.4 \%, p=0.002$, Student's two-tailed paired t-test). i, Percent reported center at each frequency relative to center stimulus on the three days prior to VNS onset (black, 'Pre') and behavior on day of maximum performance ( \pm 1 day, blue, 'VNS', $N=11$ mice; means were significantly different at -0.5 and +0.25 ; prior to VNS at $-0.5: 41.9 \pm 7.4 \%$ reported center, during VNS at $-0.5: 22.3 \pm 5.8 \%$ reported center, $p=0.005$, Student's one-tailed paired t-test; prior to VNS at $+0.25: 55.5 \pm 6.9 \%$ reported center, during VNS at $+0.25: 41.2 \pm 8.5 \%$ reported center, $p=0.03$, Student's one-tailed paired t-test ). j, Mean error rates were significantly higher prior to VNS onset relative to maximum performance at frequencies $\pm 0.25,0.5$ and 1.0 octaves from center frequency (error rate at 0 octaves/center: $-4.2 \pm 4.6 \%, p=0.18$, Student's one-tailed paired t-test; error rate at \pm 0.25 octaves from center: $-15.1 \pm 8.0 \%, p=0.04$; error rate at \pm 0.5 octaves: $-13.2 \pm 7.0 \%, p=0.04$; error rate at \pm 1 octaves: $-7.30 \pm 4.1 \%, p=0.05$; error rate at \pm 1.5 octaves: $-1.3 \pm 2.7 \%, p=0.32$ ). $\mathbf{k}$, In experimental animals ('VNS', left), performance improved at frequencies \pm 0.25 and 0.5 octaves from center during VNS (day of maximum performance \pm 1 day, blue, 'Max', $61.9 \pm 5.93 \%$ correct, mean \pm S.e.m.) relative to three days prior to VNS pairing (black, 'Pre', $47.2 \pm 5.34 \%$ correct, mean \pm s.e.m., $N=11$, Student's one-tailed paired t-test; $p=0.03$ ). In 'sham' animals (right), performance was not significantly different at frequencies \pm 0.25 and 0.5 from center prior to VNS (black, 'Pre', open circles, 39.6 $\pm 7.76 \%$, mean \pm s.e.m.) and day 11-13 of sham VNS (mean days to max performance in experimental animals, blue, open circles, $42.9 \pm 2.54 \%$, mean \pm s.e.m.) in animals with non-viable cuffs $(\mathrm{N}=6, \mathrm{p}=0.33$, Student's one-tailed paired t-test). 
period was chosen based on the time to maximum performance in the VNS animals (Fig. 2g).

As the behavioral effects of VNS pairing gradually emerged over several days, we wondered if there were also immediate perceptual effects during the paired training sessions, and/or if there were improvements occurring each day from block to block with successive training within a daily session. We first analyzed behavioral differences across blocks with and without VNS pairing, specifically comparing block one and three (without VNS) vs block two and four (with VNS). We focused on these two blocks of trials to capture potential differences, e.g., in motivational state as animals acquired more water rewards. In sessions with blocks of VNS, performance did not selectively improve during VNS pairing blocks compared to unpaired blocks, either across all stimuli (Fig. 3a; block one performance without VNS: $77.1 \pm 2.0 \%$ correct, mean \pm s.e.m.; block two performance with VNS: $73.1 \pm 2.6 \%$ correct; block three performance without VNS: $76.5 \pm 2.2 \%$ correct; block four performance with VNS: $73.8 \pm 2.5 \%$ correct; $N=11, p=0.55$, one-way ANOVA with Tukey's multiple comparisons correction), or just at frequencies $\pm 0.25-0.5$ from center (Fig. 3b; block one performance: $62.0 \pm 3.4 \%$ correct; block two performance: $58.0 \pm 4.3 \%$ correct; block three performance: $62.4 \pm 4.7 \%$ correct; block four performance: $57.7 \pm 4.5 \%$ correct; $N=11, p=0.80$, one-way ANOVA with Tukey's multiple comparisons correction). There also could be rapid changes in motivation, learning, or other aspects of animal state within a session from block to block independent of VNS pairing. To account for this, we compared performance per block in the three initial baseline sessions prior to initiating VNS pairing. We found that performance was stable across all blocks in these baseline sessions (Fig. 3c; block one performance: $71.9 \pm 2.7 \%$ correct; block two performance: $70.0 \pm 1.7 \%$ correct; block three performance: $68.7 \pm 2.5 \%$ correct; block four performance: $71.0 \pm 2.8 \% ; \mathrm{N}=11, \mathrm{p}=0.76$, one-way ANOVA with Tukey's multiple comparisons correction). Additionally, there was no significant change in response rate across blocks (Fig. 3d; block one response rate: $96.2 \pm 1.3 \%$; block two: $88.9 \pm 3.7 \%$; block three: $92.1 \pm 2.2 \%$; block four: $84.8 \pm 5.1 \%$; $N=11, p=0.10$, one-way ANOVA with Tukey's multiple comparisons correction).

Instead of immediate gains in performance during or just after VNS pairing, these results indicate that behavioral changes took days to emerge, accruing over daily sessions with VNS pairing. This would suggest that the gradual changes in performance might be observed even in block one across days, before any VNS pairing occurred on that day. To test this hypothesis, we compared performance on block one (i.e., the first 50 2AFC trials without VNS pairing of each day) to performance over all blocks across days. Animals exhibited similar rates of improvement over time for block one alone compared to performance on all five blocks (Fig. 3e,f). Across animals and days of VNS, 
performance during block one was significantly correlated with performance over all five blocks (Fig. 3g, Pearson's $\mathrm{R}=0.74, \mathrm{p}<0.001$ ). The gains in performance resulting from VNS pairing for all stimuli and just $\pm 0.25-0.5$ octaves were also evident just in the trials on block one of the best performance day (Fig. $3 \mathrm{~h}$, all stimuli, $\mathrm{N}=11$ mice, $\mathrm{p}=0.002$, Student's two-tailed paired t-test; $\pm 0.25-0.5$ octaves, $p=0.008$ ).

\section{VNS pairing enables long-term cortical plasticity}

Our results demonstrate that VNS pairing enhanced perceptual learning even beyond the limits achieved just by behavioral training. As these gains emerged gradually over days, we suspected that a mechanism related to enduring neuroplasticity was being engaged by VNS pairing. Previous studies in rats pairing pure tones with VNS 41,49 found changes to neuronal populations and tonotopic maps of primary auditory cortex (A1), strongly suggesting that auditory cortex is a locus of potentially behaviorally-relevant plasticity.

To identify possible long-term changes in cortical responses and receptive fields triggered by VNS pairing, we performed longitudinal two-photon imaging in mouse auditory cortex of seven untrained animals ( 6 wild-type females, 1 wild-type male). We injected all mice with a CaMKII-GCaMP6f in auditory cortex, waited 2-4 weeks for viral expression, and then implanted a VNS cuff electrode on the left vagus nerve. We paired a tone of a single frequency with VNS by coincidental presentation every 2.5 seconds for 5 minutes each day for at least 5 days (up to 20 days). We performed these experiments outside the context of behavior, so that we could monitor neural changes that might be more directly attributed to VNS-mediated plasticity, avoiding the confound of cortical plasticity that might occur due to training $8,9,11,12$. In each animal, we tracked a consistent population of neurons over days of pairing (Fig. 4a). We also presented pure tones of 4-64 kHz every 1-3 days throughout the pairing procedure to monitor changes across a wider frequency range of the receptive fields (Fig. 4a). Additionally, after the initial pairing session, we presented the same set of frequencies both 15 minutes and 2 hours after VNS pairing to look for more acute changes of auditory responses in a subset of animals.

We imaged from layer 2/3 excitatory neurons in each animal $(\mathrm{N}=7$ mice, $120.1 \pm 26.8$ neurons per animal, mean \pm s.d.) in a $300 \mu \mathrm{m} \times 300 \mu \mathrm{m}$ region. 391/867 neurons (45.1\%) recorded prior to VNS-tone pairing were responsive to at least one frequency between 4$64 \mathrm{kHz}$, and $371 / 815$ neurons (45.5\%) recorded on day 5 of pairing were responsive (with 465 cells imaged on both sessions). For each animal, we computed the overall best frequency across auditory cortex in the region of imaging ('local best frequency'), and 98/391 responsive neurons $(25.1 \%)$ on the first imaging day had significant or maximal responses to that local best frequency. We then chose a frequency that was initially under-represented at the level of individual neuronal tuning profiles as the 'paired 
frequency', with 49/391 responsive neurons (12.5\%) initially responding to that frequency across animals before VNS pairing.

VNS pairing modified the tuning curves of auditory-responsive cells over minutes to days, as quantified by the percentage of active neurons responding to a certain stimulus (Fig. $\mathbf{4 b , c , d ) . ~ D e s p i t e ~ o f t e n ~ o b s e r v i n g ~ l a r g e ~ i n c r e a s e s ~ i n ~ t h e ~ p a i r e d ~ f r e q u e n c y ~} 15$ minutes after pairing (Fig. 4e), these changes were generally quite transient and returned to baseline within two hours (Fig. 4f). Consistent instead with the time course of behavioral enhancement we observed with VNS, reliable and lasting cortical changes required 5+ days to emerge, but the total number of pairing days required was individually specific and still quite variable even at 5 days (Fig. $\mathbf{4 g}$ ). We focused the analysis on the day that showed the maximum change in percent of responsive neurons relative to initial tuning at the paired frequency (Fig. 4h, 5.0 \pm 3.4 days; mean \pm s.d.; $n=867$ neurons initially; $n=864$ neurons on day of max response; $\mathrm{N}=7$ mice). We measured the change in number of responsive neurons at three frequencies, the original best frequency ('BF'; open circles), the paired frequency (' $P$ ', blue), and a frequency one octave from the paired frequency (' \pm 1 ', gray) to ask how stimulus-specific were these changes. Two major changes resulted from VNS pairing: the number of neurons responding to the paired frequency significantly increased (P: $260.2 \pm 299.2 \%$ increase; mean \pm s.d., $N=7$ mice, one-way ANOVA with Tukey's multiple comparisons correction; response at paired frequency was significantly different than BF with $p=0.017$ and \pm 1 octave with $p=0.021$ ) while the number of neurons responding to the original best frequency decreased (BF: $-29.8 \pm 49.8 \%$ decrease; mean \pm s.d.). As in our past studies of the behavioral effects of pairing auditory stimuli with basal forebrain stimulation ${ }^{35}$, we hypothesize that both the changes to the paired and original best frequencies are required together, in order to effectively reshape cortical tuning curves for improving sensory perception.

\section{VNS activates cholinergic basal forebrain neurons via projections from NTS and LC}

A major outstanding question regarding the utility of VNS across species is the mechanisms of action. Specifically, it remains unclear how and where VNS leads to either direct or indirect activation of different brain regions (including auditory cortex). We reasoned that central neuromodulation - likely through the cholinergic system - was involved, given the diverse set of clinical applications of VNS in human subjects ${ }^{37-40}$, the slower rates for consistent perceptual improvement and cortical plasticity seen in mice 35,59 and the known anatomical projections from NTS ${ }^{54,55}$. However, many of the effects of VNS have been historically attributed to the titratable activation of the locus coeruleus and consequent central noradrenergic release $48,52,53,60$. The potential contribution of cholinergic modulation to VNS has been more controversial. Conventionally the 
bioRxiv preprint doi: https://doi.org/10.1101/2022.01.28.478197; this version posted Jary 28, 2022. The copyright holder for this preprint
(which was not certified by peer review) is the author/funder. All rights reserved. No reuse allowed without permission
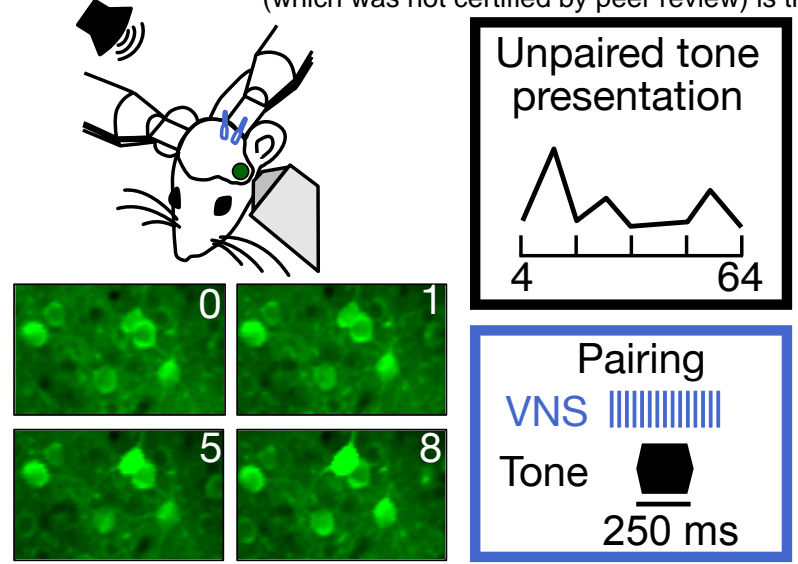

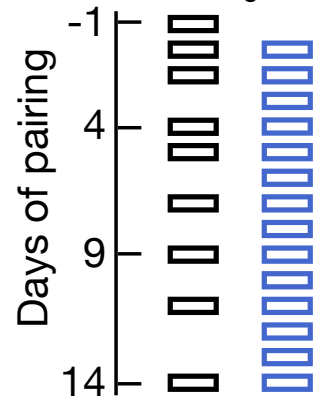

Pairing session Unpaired tone presentation

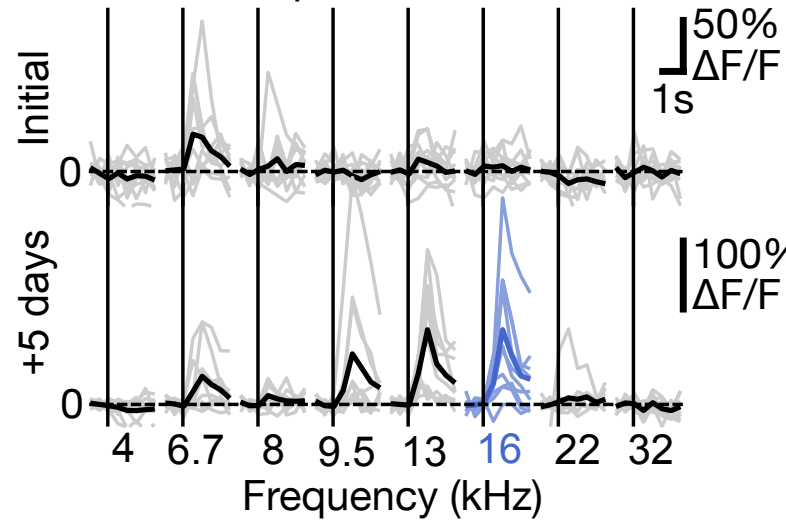

C

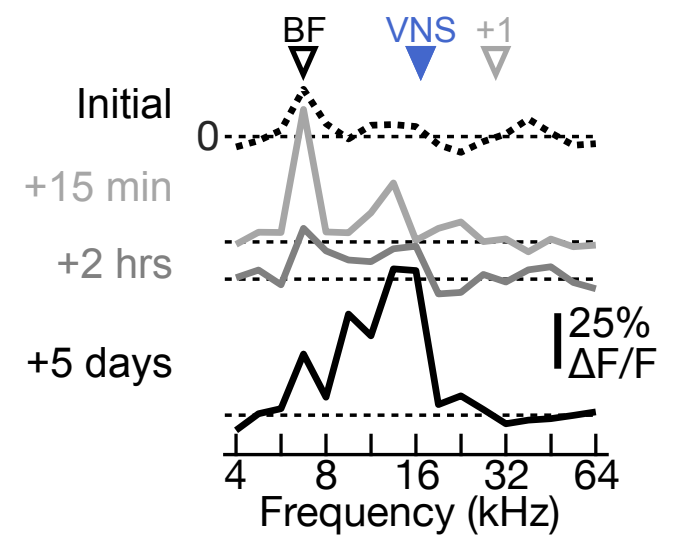

$+2 \mathrm{hrs}$

Example neuron

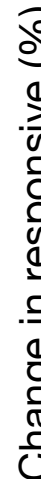

g

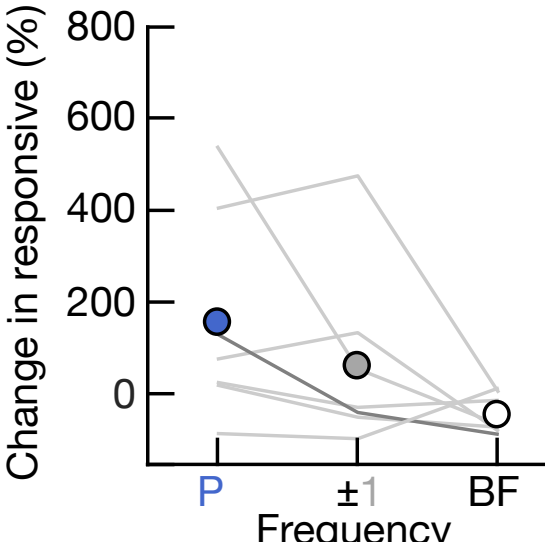

e

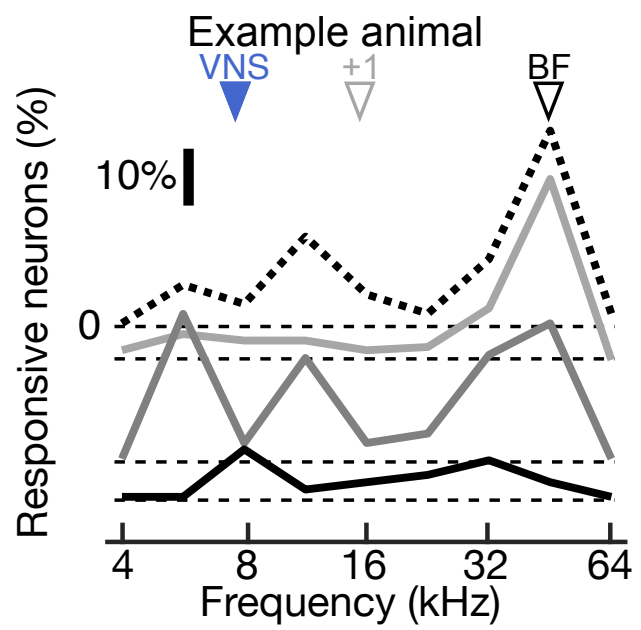

+5 days $\quad \mathbf{h}$

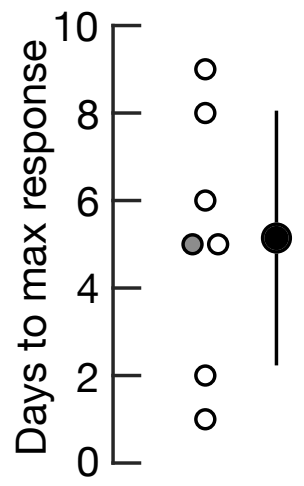

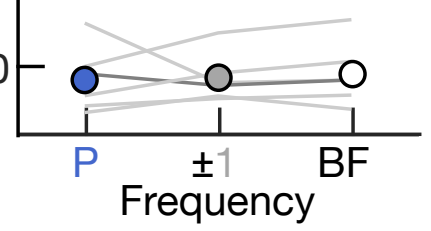

Frequency i

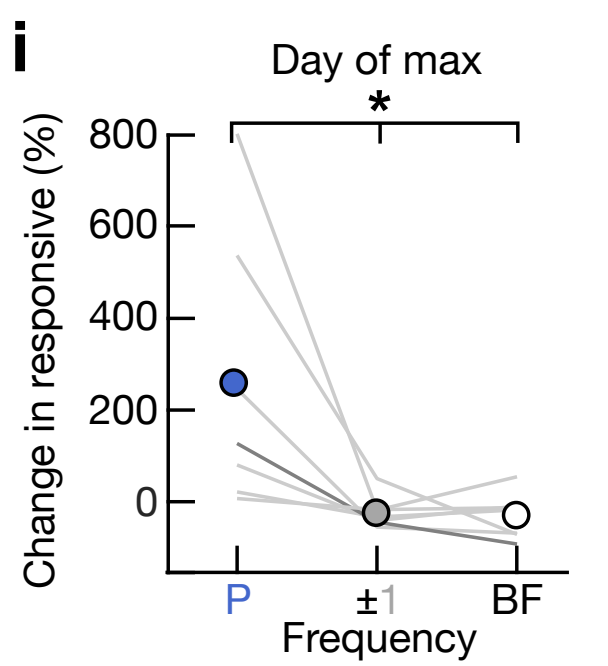

Figure 4

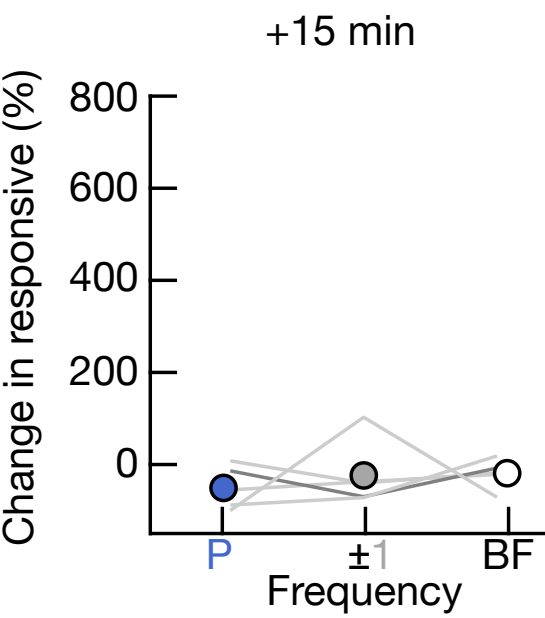


Figure 4. VNS enables long-term plasticity in excitatory neurons in auditory cortex. a, Schematic of VNS-tone pairing while performing two photon imaging of excitatory neurons in auditory cortex. During VNS-tone pairing, VNS was for $500 \mathrm{~ms}$ at $30 \mathrm{~Hz}$, centered around the $250 \mathrm{~ms}$ tone of one frequency every 2.5s. Pairing was done every day for 20 days. During unpaired, passive tone presentation, tones ranging from $4-64 \mathrm{kHz}$ at $70 \mathrm{~dB}$ are presented for $250 \mathrm{~ms}$ every 5-10s. Passive tone presentation was performed every 1-3 days. b. Example neuron during initial passive tone presentation and after 5 days of VNS-tone pairing for select frequencies. The paired tone was $16 \mathrm{kHz}$ (seen in blue post 5 days of pairing) for this animal. Mean response for each frequency is represented in black and individual trials are shown in gray. c, Passive tuning curves across all frequencies for example neuron prior to first VNS pairing (dotted line), +15 minutes post first VNS pairing (light gray), +2 hours post first VNS pairing (dark gray), and after 5 days of pairing (black). 'VNS', paired tone was $16 \mathrm{kHz}$ for this animal. ' +1 ', tone one octave from paired tone. 'BF', original best frequency across the population. d, Percentage of neurons responsive to 4 to $64 \mathrm{kHz}$ prior to VNS pairing (dotted line), +15 minutes post first pairing (light gray), +2 hours post first pairing (dark gray) and after 5 days of pairing (black) in example animal. The paired tone was 16 $\mathrm{kHz}$ for this animal. e, Change in percent responsive 15 minutes after initial VNS-tone pairing relative to initial responses at the frequency with the maximum initial response (BF; open circle; $20.0 \pm 32.6 \%$; mean \pm s.d.), paired frequency (blue; $-49.8 \pm 46.5 \%$; mean \pm s.d.) and frequency one octave away from the paired frequency (gray; $-23.2 \pm 72.3 \%$; mean \pm s.d.) ( $N=5$ mice; $p=0.63$; oneway ANOVA with Tukey's multiple comparisons correction). Example animal from $\mathbf{d}$ highlighted in dark gray. $\mathbf{f}$, Change in percent responsive 2 hours after initial VNS-tone pairing relative to initial responses at the frequency with the maximum initial response (BF; open circle; $16.8 \pm 67.8 \%$; mean \pm s.d.), paired frequency (blue; $-28.5 \pm 71.5 \%$; mean \pm s.d.) and frequency one octave away from the paired frequency (gray; $-25.3 \pm 52.1 \%$; mean \pm s.d.) $(\mathrm{N}=6$ mice; $\mathrm{p}=0.95$; one-way ANOVA with Tukey's multiple comparisons correction). Example animal from $\mathbf{d}$ highlighted in dark gray. $\mathbf{g}$, Change in percent responsive at day 5 of pairing relative to initial responses at the frequency with the maximum initial response (BF; open circle; $-46.6 \pm 42.8 \%$; mean \pm s.d.), paired frequency (blue; $155.3 \pm 226.9 \%$; mean \pm s.d.) and frequency one octave away from the paired frequency (gray; $60.6 \pm 196.3 \%$; mean \pm s.d.) ( $N=7$ mice; $p=0.13$; one-way ANOVA with Tukey's multiple comparisons correction). Example animal from $\mathbf{d}$ highlighted in dark gray. $\mathbf{h}$, Distribution of days to maximum response at paired frequency $(5.1 \pm 2.9$ days; mean \pm s.d.; $N=7$ mice). Gray circle is animal represented in $\mathbf{d}$. $\mathbf{i}$, Change in percent responsive at day of maximum population response to paired frequency relative to initial response at frequency with the maximum initial response (BF; open circle; $-29.8 \pm 49.8 \%$; mean \pm s.d.), paired frequency (blue, $260.2 \pm 299.2 \%$; mean \pm s.d.) and frequency one octave away from paired frequency $(-21.4 \pm 34.9 \%$; mean \pm s.d.) ( $N=7$ mice; one-way ANOVA with Tukey's multiple comparisons correction; response at paired frequency is significantly different than BF and \pm 1 octave ( $p=0.017, p=0.021$, respectively). Day of maximum population response was determined for individual animals in $\mathbf{h}$. Example animal from $\mathbf{d}$ highlighted in dark gray. 
cholinergic basal forebrain was thought to receive only indirect input from NTS via locus coeruleus ${ }^{54,55}$. While behavioral evidence links motor learning with the recruitment of cholinergic basal forebrain neurons via VNS ${ }^{46,61}$, there are differences in the functional organization of cholinergic inputs to various cortical regions ${ }^{30,62-65}$. Thus, we next wanted to establish if the central cholinergic system might contribute to the sensory perceptual improvements we observed with VNS.

We performed retrograde tracing studies from cholinergic neurons in basal forebrain using a Cre-inducible, retrograde pseudotyped monosynaptic rabies virus ${ }^{66,67}$. We targeted cholinergic neurons by injecting pAAV-TREtight-mTagBFP2-B19G and pAAVsyn-FLEX-splitTVA-EGFP-tTA in the basal forebrain of ChAT-Cre mice $(\mathrm{N}=3)$ followed by an injection of SAD $\triangle$ G-mCherry two weeks later (Fig. 5a). We found mCherry expression in both NTS and locus coeruleus, indicating that basal forebrain cholinergic neurons receive direct input from both areas (Fig. 5b,c). To reveal the cell types in NTS and locus coeruleus that projected to basal forebrain, in other animals $(\mathrm{N}=4)$ we also injected a Credependent tdTomato with a retrograde promoter in basal forebrain of TH-Cre animals (Fig. 5d). We saw tdTomato expression in both locus coeruleus and NTS (Fig. 5e,f). These anatomical studies show that cholinergic basal forebrain neurons receive input from $\mathrm{TH}+$ neurons in both locus coeruleus and NTS (Fig. 5g).

We next asked if these direct and indirect inputs to basal forebrain cholinergic cells were functionally activated by VNS. We used two-photon imaging of cholinergic axon fibers in auditory cortex (Fig. 5h) to more specifically monitor recruitment of cortically-projecting cholinergic inputs that might produce the behavioral and neuronal effects of VNS tone pairing described above. Some animals used for axon imaging were ChAT-Cre mice injected with Cre-dependent GCaMP6s in basal forebrain $(\mathrm{N}=3)$; other animals were transgenic ChAT-Cre mice crossed with Ai162 (transgenic GCaMP6s) mice with tdTomato injected into basal forebrain $(\mathrm{N}=3)$. We found that VNS strongly activated many cholinergic axon fibers in auditory cortex (Fig. 5i,j). Therefore, VNS activates auditory cortical projecting cholinergic neurons in basal forebrain via input from both locus coeruleus and NTS.

\section{Optogenetic manipulation of the central cholinergic system and VNS}

In the final set of experiments, given the activation of cholinergic fibers in auditory cortex, we asked how cholinergic modulation related to VNS pairing in behaving animals. Since areas of cortex receive input from distinct subsets of cholinergic neurons ${ }^{62,63}$, we aimed to target only the auditory cortical-projecting cholinergic neurons. For projection-specific opsin expression, we bilaterally injected a retrograde, Cre-dependent channelrhodopsin2 (ChR2) virus in auditory cortex and implanted optic fibers above basal forebrain in five 
1) Mix of split AwAich was not-

FLEX-GFP

2) $\triangle \mathrm{G}-\mathrm{RV}$-mCherry

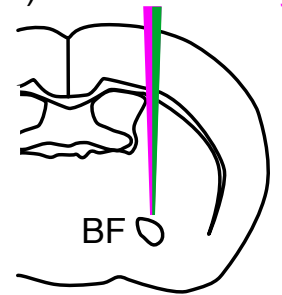

ChAT-cre
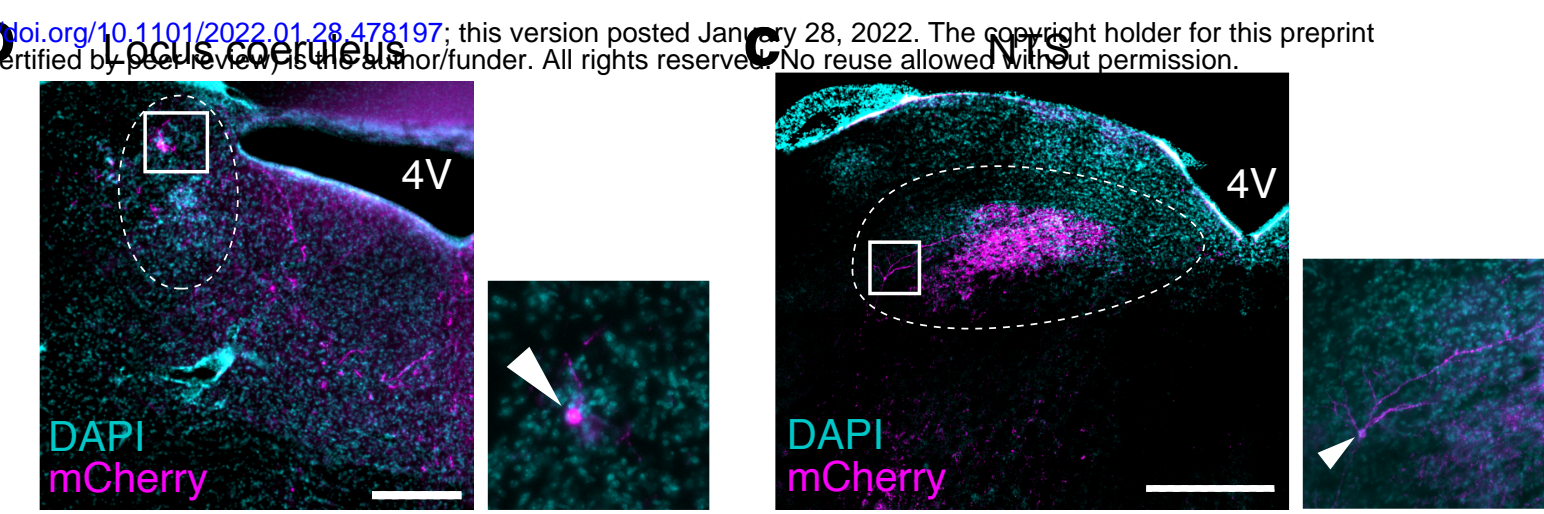

$\mathbf{e}$

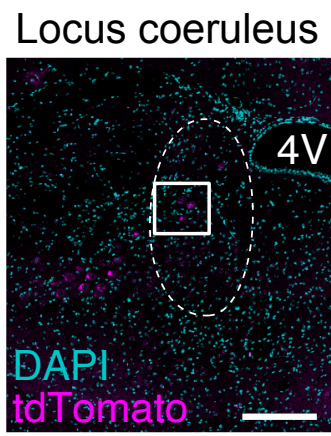

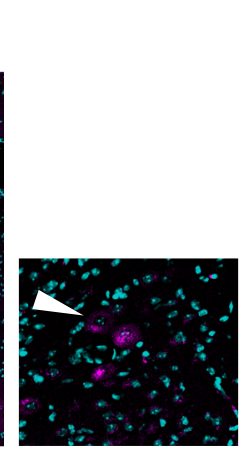
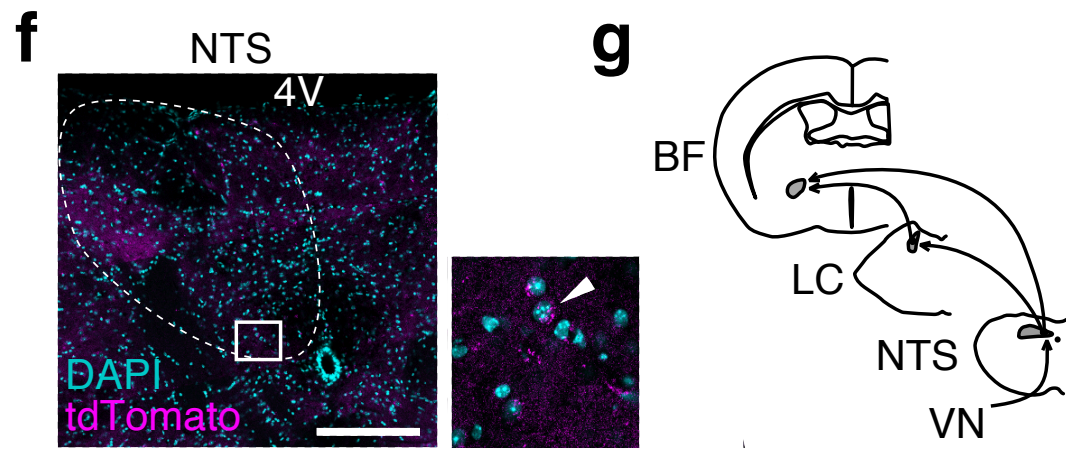

TH-Cre

i

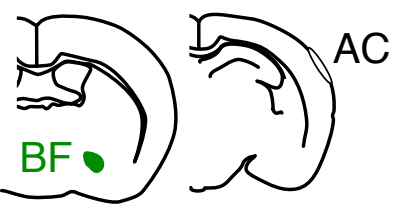

ChAT-Cre

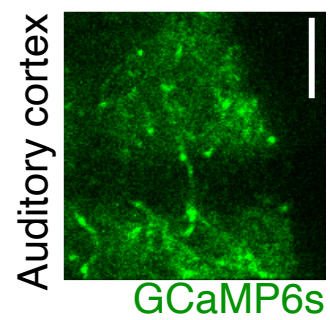

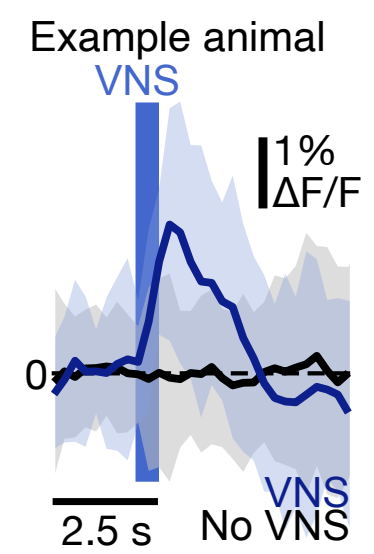

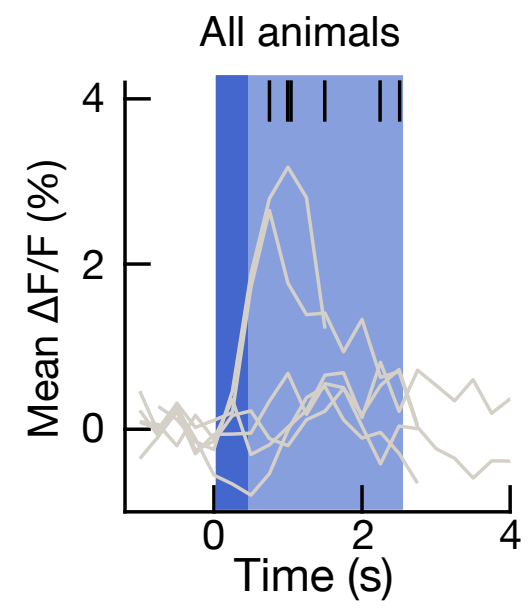

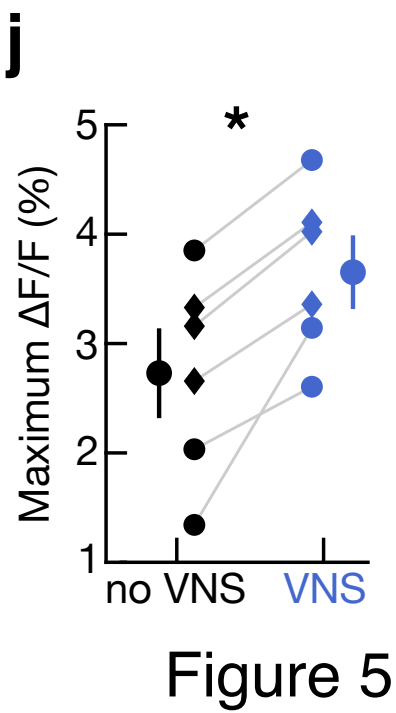

Figure 5. Stimulating the vagus nerve activates cholinergic basal forebrain via inputs from NTS and locus coeruleus. a, Schematic for mapping inputs to cholinergic basal forebrain neurons using retrograde, Cre-dependent, pseudotyped monosynaptic rabies. b, mCherry expression in locus coeruleus. Scale bar is $500 \mu \mathrm{m}$. c, mCherry expression in nucleus tractus solitarus (NTS). Scale bar is $500 \mu \mathrm{m}$. d, Schematic of injection of retrograde Cre-dependent tdTomato into basal forebrain of TH-Cre mice. e, tdTomato positive neurons in locus coeruleus. Scale bar is $200 \mu \mathrm{m}$. $\mathbf{f}$, tdTomato positive neurons in NTS. Scale bar is $250 \mu \mathrm{m}$. $\mathbf{g}$, Schematic of proposed anatomical connections between NTS, locus coeruleus and basal forebrain. $\mathbf{h}$, Schematic for two photon imaging of cholinergic axons from basal forebrain in auditory cortex. Example imaging region from one animal. Scale bar is $50 \mu \mathrm{m}$. $\mathbf{i}$, Average VNS evoked response in cholinergic axons. Mean response of cholinergic BF axons to VNS (blue) or during spontaneous activity (black) in an example animal ( $\mathrm{n}=11$ trials, left). Mean response of cholinergic BF axons in auditory cortex to VNS with an intensity of $0.8 \mathrm{~mA}$ ( $\mathrm{N}=6$ animals, right). Black tick marks represent time of maximum response for each animal. j, VNS elicits significant response in cholinergic axons in auditory cortex. Mean maximum response in the 1.5s during and immediately following $500 \mathrm{~ms}$ of VNS (blue; $3.65 \pm 0.37 \% \Delta \mathrm{F} / \mathrm{F}$; mean \pm S.e.m.) or in sessions without VNS (black; $2.73 \pm 0.41 \% \Delta \mathrm{F} / \mathrm{F}$; mean \pm s.e.m., $\mathrm{N}=6$ mice; $11-60$ trials of VNS per animal; Student's two-tailed paired t-test; $p=0.004$; circles represent viral injection of GCaMP6s in basal forebrain of ChAT-Cre, diamonds represent transgenic GCaMP6s in ChAT-Cre mice). 
ChAT-Cre animals trained on the 2AFC task from Figure 1 (Fig. 6a). After 9+ days in stage three, instead of VNS pairing (as these animals were not cuffed), we optogenetically stimulated auditory cortical-projecting cholinergic neurons in the same manner as VNS: $500 \mathrm{~ms}$ duration, $30 \mathrm{~Hz}$ pulse rate, centered around the $250 \mathrm{~ms}$ tone during training blocks two and four.

We found that optogenetic activation of cholinergic neurons during behavior led to similar improvements in perceptual learning as with VNS pairing. The maximum change in 2AFC task performance occurred several days after optogenetic pairing (Fig. 6b,c, best performance after $9.8 \pm 3.0$ days, $\mathrm{N}=5$ mice, mean \pm s.d.). We compared behavioral performance on the best day from optogenetic pairing ( \pm 1 day, to include the day before and after the best day for each animal, averaging over those three days) to three days of baseline behavioral performance. Maximum performance was significantly increased past the level obtained just from days of stage three training (Fig. $\mathbf{6 d}$, before optogenetic pairing: $66.1 \pm 2.6 \%$ correct over all center/non-center stimuli, best day after pairing: $73.2 \pm 2.6 \%$ correct over all stimuli, $\mathrm{N}=5$ mice, $\mathrm{p}=0.03$, Student's two-tailed paired t-test). Performance gains occurred at specific non-center flanking frequencies as with VNS pairing, specifically at $\pm 0.5-1.5$ octaves from center (Fig. $6 e, f$, change in error at \pm 0.5 octaves from center: $-15.1 \pm 3.2 \%, p=0.014$, Student's one-tailed paired t-test; change in error at \pm 1.0 octaves: $-17.0 \pm 4.4 \%, p=0.026$; change in error at \pm 1.5 octave: $-15.4 \pm 2.2 \%$, $\mathrm{p}=0.037$; behavior of individual animals shown in Extended Data Fig. 3).

While the decrease in error rates with optogenetic pairing occurred over a broader range of stimulus frequencies than VNS pairing, the time course and specific reductions to offcenter flanking tones was a consistent feature of both pairing methods. These observations support the hypothesis that the predominant effects of VNS pairing for this 2AFC task in mice is through cholinergic recruitment. To test this hypothesis more directly, we optogenetically suppressed the activity of cholinergic basal forebrain neurons during VNS pairing (Fig. 6g). In other trained ChAT-Cre animals from Figure 1 ( $\mathrm{N}=7$ mice), we bilaterally injected a Cre-dependent inhibitory opsin (archaerhodopsin) in the basal forebrain, implanted optic fibers bilaterally over basal forebrain, and implanted a cuff electrode on the left vagus nerve. After 9+ days of stage three training, we began VNS pairing as before (performing VNS on each training day during blocks two and four). During VNS pairing blocks, we concurrently optogenetically suppressed ChAT+ basal forebrain neurons continuously for $500 \mathrm{msec}$ starting $125 \mathrm{~ms}$ before tone onset. Optogenetic suppression of basal forebrain cholinergic neurons completely prevented behavioral gains from VNS pairing, both to overall performance (Fig. $6 \mathbf{h , i}, \mathrm{N}=7$ mice, before VNS and optogenetic suppression: $62.7 \pm 2.2 \%$ correct over all center/non-center stimuli, day $11-13$ of VNS and optogenetic suppression: $61.6 \pm 2.1 \%$ correct over all 
Figure 6. Activating auditory cortical projecting cholinergic basal forebrain neurons improves perceptual performance. a, Schematic of optogenetic activation of auditory cortical projecting cholinergic BF neurons during behavior. Auditory cortical projecting cholinergic BF neurons were targeted using a retrograde Cre-dependent channelrhodopsin injected into auditory cortex of ChAT-Cre mice. ChAT+ BF activation was applied in the same blockwise fashion as previously described for $500 \mathrm{~ms}$ at $30 \mathrm{~Hz}$ centered around the tones. b. Optogenetic activation of $\mathrm{ChAT}+\mathrm{BF}$ neurons during behavior gradually improved performance over days ( $\mathrm{N}=5$ mice). c, Days to maximum performance per animal after initiation of optogenetic pairing during behavior $(9.8 \pm 3.0$ days, mean \pm s.d.). d, Performance over all stimuli improves after optogenetic pairing. Percent correct on day with maximum performance $( \pm 1$ day) of VNS pairing across all frequencies (light blue; $73.2 \pm 2.6 \%$, mean \pm s.e.m., $\mathrm{N}=5$ ) in comparison to the behavior three days prior to VNS (black; $66.1 \pm 2.6 \%, p=0.03$, Student's two-tailed paired t-test). e, Percent reported center at each frequency relative to center stimulus on the three days prior to optogenetic pairing onset (black) and behavior on day of maximum performance ( \pm 1 day; light blue, $\mathrm{N}=5$ mice). $f$, Mean error rates were significantly higher prior to optogenetic pairing onset relative to maximum performance at frequencies $\pm 0.5,1.0$ and 1.5 octaves from center frequency (difference in error rate at 0 octaves/center: $-0.4 \pm 2.2 \%, p=0.45$, Student's one-tailed paired t-test; difference in error rate at \pm 0.25 octaves from center: $-0.1 \pm 1.8 \%, p=0.49$; difference in error rate at \pm 0.5 octaves: $-15.1 \pm 3.2 \%$, $p=0.01$; difference in error rate at \pm 1 octaves: $-17.0 \pm 4.4 \%, p=0.03$; difference in error rate at \pm 1.5 octaves: $-15.4 \pm 2.2 \%, p=0.004)$. $g$, Schematic of VNS and optogenetic inhibition of cholinergic BF neurons during behavior. VNS was applied in the same blockwise fashion as previously described. Cholinergic BF neurons were optogenetically inhibited using a Cre-dependent Archaerhodopsin and green light $(565 \mathrm{~nm})$ for the entire duration of VNS during behavior (500 ms). h, Optogenetic inhibition of ChAT+ BF neurons during VNS during behavior abolishes improved performance $(\mathrm{N}=7$ mice). i, Performance did not improve after optogenetic inhibition during VNS. Percent correct on day 11-13 of optogenetic inhibition during VNS (mean day of maximum improvement in VNS paired animals; yellow; $61.6 \pm 2.1 \%$, mean \pm s.e.m., $N=7$ ) in comparison to the behavior three days prior to VNS (black; $62.7 \pm 2.2 \%, p=0.43$, Student's two-tailed paired t-test). j, Percent reported center at each frequency relative to center stimulus on the three days prior to start of optogenetic inhibition with VNS pairing onset (black) and behavior on day of 11-13 (yellow, $\mathrm{N}=7$ mice). $\mathbf{k}$, Mean error rates were not significantly different prior to optogenetic inhibition during VNS pairing onset (difference in error rate at 0 octaves/center: $-6.7 \pm 4.3 \%, p=0.24$, Student's one-tailed paired t-test; difference in error rate at \pm 0.25 octaves from center: $-7.0 \pm 3.3 \%, p=0.13$; difference in error rate at \pm 0.5 octaves: $-6.1 \pm 3.2 \%, p=0.16$; difference in error rate at \pm 1 octaves: $-0.6 \pm 4.1 \%, p=0.90$; difference in error rate at \pm 1.5 octaves: $-2.2 \pm 2.4 \%, p=0.48$ ). I, Performance at frequencies \pm 0.25 and \pm 0.5 from center did not improve after optogenetic inhibition during VNS. Percent correct on day 11-13 of optogenetic inhibition during VNS (mean day of maximum improvement in VNS paired animals; yellow; $37.8 \pm 7.0 \%$, mean \pm s.e.m., $N=7$ ) in comparison to the behavior three days prior to VNS (black; $31.1 \pm 6.7 \%, p=0.11$, Student's two-tailed paired t-test). 
stimuli, $p=0.43$, Student's two-tailed paired t-test), and in terms of error rates across specific center and non-center stimuli (Fig. 6j,k,l, change in error at 0 octaves from center: $6.7 \pm 4.3 \%, p=0.88$, Student's one-tailed paired t-test; change in error at \pm 0.25 octaves: $7.0 \pm 3.3 \%, p=0.06$; change in error at \pm 0.5 octaves: $-6.1 \pm 3.2 \%, p=0.079$; change in error at \pm 1.0 octaves: $-0.6 \pm 4.1 \%, p=0.45$; change in error at \pm 1.5 octave: $-2.2 \pm 2.4 \%, p=0.24$; behavior of individual animals shown in Extended Data Fig. 4). Collectively our results highlight the importance of the central cholinergic modulatory system for enhancements of perceptual learning via VNS.

\section{Discussion}

Although the central nervous system is highly plastic, there are limitations on the extent of changes induced by different experiences or mechanisms. For the auditory 2AFC task we used here, animals made the most errors within half an octave of the 'lick left' reference tone; VNS pairing could reduce error rates and sharpen behavioral performance, albeit not completely. Some limits in terms of plasticity and behavioral improvement are set by the physical properties of the sensory epithelium. In the visual domain, this includes retinal photoreceptor density and single-photon sensitivity68,69; for the auditory system, the ability to resolve different frequencies is constrained by the biophysics of the cochlear membrane and hair cell dynamics ${ }^{56,57}$. These properties are likely to provide hard bounds on perceptual resolution and are difficult to overcome by alternative mechanisms for potential plasticity.

Other limitations, however, might be due to other factors such as motivational state, behavioral engagement, and/or the understanding of task rules and variables. These other factors likely reflect the activation (or lack thereof) of central modulatory systems including the cholinergic basal forebrain ${ }^{31,70,71}$. Indeed, here we found that bidirectional regulation of cholinergic modulation could affect $2 \mathrm{AFC}$ task performance, with activation of cholinergic neurons mimicking the effects of VNS on enhancing task performance, whereas suppressing cholinergic neuron activity blocked the effects of VNS. Similarly, we and others have previously shown that engagement in similar auditory ${ }^{29-31,65}$ or visual ${ }^{27}$ tasks activates neurons of the cholinergic basal forebrain, that cholinergic modulation is important for task performance 28,31 , and that artificially enhancing cholinergic modulation (via pharmacology or electrical/optogenetic stimulation) can boost task performance past the levels achieved purely by behavioral training $24,28,31,34,35$. It remains unclear why the cholinergic basal forebrain is not normally fully engaged during task performance. As the effects of VNS on task performance were weakest for the best-performing animals, it is possible that the degree of cholinergic activation is a major predictor of individual sensory processing abilities and perceptual learning rates. 
The vagus nerve is remarkably complex, connecting to with several peripheral organs to provide a multiplexed input to the brainstem NTS ${ }^{72}$. In turn, the NTS sends projections to several regions important for central neuromodulation, including the oxytocin system of the hypothalamus ${ }^{73}$, the noradrenergic locus coeruleus ${ }^{54,55,73}$, and the cholinergic basal forebrain ${ }^{62-64}$. Potentially any or all of these systems might be activated by VNS, although little is known how patterns of vagal activation (either naturally occurring or via VNS) lead to similar or differential recruitment of these diverse modulatory systems. The specific parameters used for VNS (e.g., stimulation rate or intensity) might also lead to variability in terms of which downstream systems are reliably activated. We used here a consensus VNS parameter set found to generally be effective across a number of different outcome measures and species ${ }^{41,45-48}$, but there could be other stimulation regimes or longer-term dynamics that might shift the net effects of VNS. There may also be species-specificity in terms of central consequences of VNS. While aspects of the neuroanatomical organization of the vagus and NTS seem largely conserved, the thickness and composition of the vagus nerve bundle is considerably different across species, which would result in different subsets of fibers being activated by electrical stimulation ${ }^{74,75}$.

Despite this complexity, it appeared that VNS as used here in mice largely resulted in cholinergic modulation of auditory cortex. Although NTS projects to locus coeruleus ${ }^{54}$ and locus coeruleus projects to basal forebrain ${ }^{55}$, our anatomical tracing studies also revealed direct projections from both the locus coeruleus and NTS to the basal forebrain, indicating that the cholinergic system might be a major point of convergence for vagal inputs from the brainstem to affect cortical function and behavior. Previous studies have shown that enhancement of perceptual learning and behavioral performance with cholinergic modulation can be surprisingly slow, taking days to weeks to emerge ${ }^{24,59}$. This is similar to the gradual improvements in 2AFC performance we observed here with VNS pairing. In contrast, the effects of locus coeruleus pairing can be much more rapid, but possibly at the expense of initial performance ${ }^{59}$, and over-activation of the locus coeruleus can cause behavioral arrest $^{76}$. If these systems are somewhat in opposition, especially for their short-term effects, it is possible that combined stimulation of multiple modulatory centers leads to no net improvement in the moment. This might account for why we observed essentially no effects of VNS stimulation during or immediately after paired blocks, but instead that the results of VNS pairing required days to be observed across animals. The relatively slow expression of changes after VNS pairing might reflect a sleep-dependent process requiring days for consolidation, and/or plasticity within neuromodulatory areas such as the basal forebrain than then impact enhanced functionality only after reaching some threshold level of modification. 
Neuroprosthetic devices can provide successful treatments for a wide range of debilitating conditions, and perhaps could be adopted for use in augmenting performance outside of clinical care. Some types of devices are implanted centrally, such as deep brain stimulation electrodes, temporal lobe electrodes for regulating seizures, or motor cortex implants in cases of tetraplegia. The invasive nature of central implants limits their utility to only the most severe conditions. In contrast, more human subjects have received neuroprosthetic implants for peripheral nerve stimulation; e.g., cochlear implants have been used in over 500,000 people and are largely successful in terms of hearing restoration. Vagus nerve cuffs have been used in over 100,000 human subjects, mainly for treatment of epilepsy as well as other conditions ${ }^{38}$. A new generation of less-invasive or non-invasive peripheral nerve stimulators targeting the hypoglossal nerve or the auricular branch of the vagus nerve may be promising in terms of efficacy, although much more work is required to validate these devices and determine optimal stimulation regimes $^{77}$. Our data suggest that some outcomes of successful VNS might take days, weeks, or even longer to be revealed, and our results provide a potential mechanistic basis by which VNS can enhance auditory perceptual learning. 


\section{Methods \\ Animals}

All procedures were approved under an NYU Langone Institutional Animal Care and Use Committee protocol. Male and female mice aged 6-20 weeks old were used in all experiments (Fig. 1: $\mathrm{N}=29,19$ male, 10 female; Fig. 2: $\mathrm{N}=17,12$ male, 5 female; Fig. 3: $\mathrm{N}=11$, 8 male, 3 female; Fig. 4: $\mathrm{N}=8$, 1 male, 7 female; Fig. 5. $\mathrm{N}=13,6$ male, 7 female; Fig. 6: $\mathrm{N}=12,7$ male, 5 female). Genotypes used were wild-type C57BL/6J (The Jackson Laboratory, Stock No: 000664), ChAT-Cre (The Jackson Laboratory, Stock No: 028861), TH-Cre (The Jackson Laboratory, Stock No: 008601), and Ai162D (The Jackson Laboratory, Stock No: 031562). All mice had a C57BL/6J background. Mice were housed in a temperature and humidity controlled room maintained on a 12 hour light/dark cycle. Animals used in behavior were given $1 \mathrm{~mL}$ water/day. If their weight dropped below $80 \%$ of original, they were given ad libitum water until weight returned to $\geq 80 \%$ original value.

\section{AFC Behavioral Training}

Behavioral events (lick detection, auditory stimulus delivery, water reward delivery) were monitored and controlled by custom MATLAB programs interfacing with an RZ6 auditory digital signal processor (Tucker-Davis Technologies) via RPvdsEx software (TuckerDavis Technologies). Licks were detected using capacitance sensors (SparkFun, Part number: AT42QT1011) and water was delivered using solenoids (The Lee Company, Part number: LHDA0581215H). Animals were restrained using custom headposts (Ponoko).

Behavioral training on the auditory 2AFC task began after 7+ days of water restriction. Training started with habituation to head-fixation with water delivered to the mouse while it sat in a plexiglass tube. This was followed by lick port sampling sessions, in which the animal could receive water by alternating licking between the two ports with a minimum of 3 seconds between possible rewards. Mice typically learned to alternate ports while licking for 2-4 $\mu \mathrm{L}$ water droplets in 2-4 sessions. Once animals reliably licked to receive water from lick ports, stage 1 training was begun (i.e., animals were trained to lick left for the center frequency and lick right for one non-center frequency). The center frequency was chosen to be either $11.3,13.4$, or $16 \mathrm{kHz}$ (each animal had a single consistent center frequency pseudo-randomly selected from those three values). Noncenter frequencies were set per animal to be $\pm 0.25, \pm 0.5, \pm 1.0$, and \pm 1.5 octaves from the selected center frequency. In stage 1 , the only non-center frequency was either +1.5 octaves or -1.5 octaves from center (and whether higher or lower frequency was also pseudo-randomly assigned per animal).

In stage 1, while an animal's performance remained $<80 \%$ correct, they were rewarded with water regardless of behavior choice on $15 \%$ of trials to help promote consistent licking during training. Once performance reached $\geq 80 \%$ correct for three consecutive days in stage 1, animals moved to stage 2 in which the other non-center 
frequency (either \pm 1.5 octaves away) was added. After three days in stage 2 , animals moved to stage 3 regardless of performance (in which all other non-center stimuli \pm 0.25 , \pm 0.5 , and \pm 1.0 , octaves from the center frequency were also presented and rewarded for right-side licking).

On each trial, a 250 ms tone was presented and animals had to classify the tone as the center frequency (green) or any other frequency (shades of gray). Stimuli were presented at $70 \mathrm{~dB}$ SPL in a pseudorandom order, such that the likelihood of center:noncenter was 1:1 (with frequency uniformly chosen from the non-center distribution on noncenter trials). After a $250 \mathrm{~ms}$ delay, animals had to lick left to report the stimulus as 'center' and had to lick right to report the stimulus as 'non-center'. If the animal did not respond during the 2.5 seconds of the response epoch, the trial was classified as a 'no response' trial (which were excluded from analysis except where otherwise noted). If the lick response was correct, a small water reward (2-4 $\mu \mathrm{L}$ ) was delivered to the corresponding lick port. Inter-trial intervals were $3 \pm 0.5$ seconds (mean \pm s.d.) on trials with a correct response and $6 \pm 0.5$ seconds (mean \pm s.d.) on trials with an incorrect response or without a response. Animals were not punished for licking outside of the response epoch. Animals generally performed between 350-500 trials/day.

\section{Vagus nerve stimulation in mice}

The custom cuff electrode design was adapted for mice from a previous cuff electrode designed for rats ${ }^{41,42}$. A bipolar stimulating peripheral nerve cuff electrode was custom built using micro-renathane tubing (Braintree Scientific, Part number: MRE-040), coated platinum iridium wire (Medwire, Part number: 10IR9/49T), and gold pins (Mouser Electronics, Part number: 575-100140). The tubing was used as the base for the portion of the cuff that interacts with the nerve. It was cut in 1.0-1.5 mm segments, with an interior diameter of 0.025 inches to allow for nerve swelling after implantation. Two platinum iridium wires with the coating removed were glued (Dentsply Sirona, Triad gel) to the interior of the tubing about $0.5 \mathrm{~mm}$ apart. Gold pins were soldered to the end of the wires opposite the tubing. The coating on the wire was only removed for small portions at both ends to limit non-coated contact only to the gold pins and within the interior circumference of the tubing. Wires were $2.5-3.0 \mathrm{~cm}$ in length in order to span from the skull to the position of the cervical vagus. Non-absorbable silk suture string (Braintree Scientific, Part number: SUT-S 104) was added to each side of the cuff opening to improve cuff handling and manipulation during implantation. Viable cuff electrodes were determined by an impedance reading between $1-10 \mathrm{k} \Omega$ at $1 \mathrm{kHz}$ when submerged in saline (Peak Instruments LCR45).

Impedance measurements were taken each day of stimulation (Peak Instruments LCR45, Frequency: $1 \mathrm{kHz}$ ). Measurements of breathing rates, $\mathrm{SpO}_{2}$, and heart rates were collected using a thigh sensor (MouseOx) in animals lightly anesthetized with $0.75-1.5 \%$ 
isoflurane during VNS. The vagus nerve was stimulated using a high-current stimulus isolator (World Precision Instruments A385) triggered by a digital signal processor (RZ6, Tucker-Davis Technologies). VNS parameters were based on previous work ${ }^{41,46-48: 100}$ $\mu$ s pulse width, $30 \mathrm{~Hz}$ stimulation rate, 0.5 second duration. Stimulation intensity was 0.6 $0.8 \mathrm{~mA}$, based on the magnitude of the effect on vitals (VNS intensity was $0.8 \mathrm{~mA}$ for imaging studies of Figs. 4,5).

Surgeries were performed on mice aged 6-12 weeks. Mice were anesthetized using isoflurane (1.0-2.5\%) and positioned in a stereotaxic frame (Kopf, model 923-B). Body temperature was maintained at $37^{\circ} \mathrm{C}$ with a heating pad and rectal temperature probe. For behavior and imaging experiments, a custom headpost ${ }^{31}$ was attached to the skull using dental cement (C\&B-metabond), after thorough cleaning using hydrogen peroxide. Following all surgical procedures, animals were given a nutritionally-fortified water gel for recovery assistance (Clear $\mathrm{H}_{2} \mathrm{O}$ DietGel Recovery, Part number: 72-065022).

For cuff implantation, a magnetic retractor base plate (Fine Science Tools, Part number: 1800-03) was used instead of a stereotaxic frame to allow for more flexible positioning of the animal. Mice were positioned semi-supine at a $45^{\circ}$ angel on their right side. Hair on the chest was removed with Nair from the sternum to the left shoulder. The surgical site was sterilized by alternating $70 \%$ ethanol and betadine washes. A $1.5 \mathrm{~cm}$ incision was made $0.5-1.0 \mathrm{~cm}$ to the left of the top of the sternum, then the submandibular gland was separated and retracted from connective tissue. Using blunt forceps (Fine Science Tools, Part number: 11231-30), the left sternocleidomastoid and omohyoid muscles were separated and retracted until the carotid sheath was accessible. A sterilized cuff electrode was led subcutaneously from the left side of the scalp incision, between the ear and eye, and down to the chest incision site. Once positioned, the cuff end of the electrode was deposited near the carotid sheath and the gold pin leads remained exposed on the head. Using sharp forceps (Fine Science Tools, Part number: 11251-30), a $4 \mathrm{~mm}$ stretch of the cervical vagus nerve was isolated from surrounding nerves and vasculature without direct contact with the nerve. The cuff electrode was positioned around the vagus nerve so the nerve was not taut and the non-coated intra-cuff wires had even contact with the nerve. The cuff was knotted closed with non-absorbable silk suture string and muscles were returned to their original positions. Absorbable sutures (Ethicon, Part number: W1621T) were occasionally made on the muscles to keep the cuff from lifting the nerve ventrally. The submandibular gland was repositioned and the skin was sutured closed with absorbable sutures (Ethicon, Part number: VCP433). The sutures were sterilized with betadine and then sealed with surgical glue (Meridian). The electrode cuff leads were secured near the headpost using dental cement (C\&B-metabond). The incision was covered with $4 \%$ topical lidocaine (L.M.X. 4). Cuff electrode impedance (1 kHz) was recorded immediately after surgery and maintained a similar measurement from before 
implantation. Successful cuff implantation was verified using both impedance measurements and changes in vitals readings as described above.

All VNS during behavior started after the animals reached stable performance after a minimum of nine days from start of stage three. All animals in either the VNS or sham group received 17-20 days of stimulation. On each day of stimulation, animals performed a total of 400 trials, with 100 trials (two blocks of 50 trials, blocks two and four) of behavior with VNS. All auditory stimuli were stimulated to avoid the animal using solely VNS to identify specific stimuli. VNS lasted 500 ms and was centered around the tone, i.e., it started $125 \mathrm{~ms}$ prior to tone onset and ended $125 \mathrm{~ms}$ after tone offset. Stimulation days ended with 100 trials of unstimulated behavior.

\section{Two-photon calcium imaging}

Cranial window implantation over left auditory cortex was performed, as previously described $^{31}$. For cell body imaging, $1.0 \mu \mathrm{L}$ of diluted CaMKII.GCaMP6f (AAV1, diluted 1:3 with dPBS or AAV9, diluted 1:10 with dPBS, Addgene number: 100834) was injected into auditory cortex (1.5 mm from lambda, along lateral suture). For axon imaging, either 1.0 $\mu \mathrm{L}$ of AAV1.pCAG.FLEX.tdTomato.WPRE (Addgene number: 51503) was injected in basal forebrain (AP: $-0.5 \mathrm{~mm}, \mathrm{ML}$ : $-1.8 \mathrm{~mm}$, DV: $-4.5 \mathrm{~mm}$ from brain surface) of heterozygous ChAT-Cre and Ai162D mice or $1.0 \mu \mathrm{L}$ of pAAV.Syn.Flex.GCaMP6s.WPRE.SV40 (diluted 1:2 in dPBS; Addgene: 100845-AAV5) was injected in basal forebrain of heterozygous ChAT-Cre mice.

Two-photon fluorescence of GCaMP6f/s and tdTomato was excited at $900 \mathrm{~nm}$ using a mode locked Ti:Sapphire laser (MaiTai, Spectra-Physics, Mountain View, CA) and detected in the green channel and red channel, respectively. Imaging was performed on a multiphoton imaging system (Moveable Objective Microscope, Sutter Instruments) equipped with a water immersion objective (20X, NA=0.95, Olympus) and the emission path was shielded from external light contamination. Images were collected using Scanlmage (Janelia). To image auditory cortex, the objective was tilted to an angle of 50$60^{\circ}$.

Awake animals were head-fixed under the microscope and the stimulus isolator was connected to the VNS cuff. We imaged $\sim 300 \mu \mathrm{m}^{2}$ areas in auditory cortex (scan rate $\sim 4 \mathrm{~Hz}, 0.26 \mathrm{~s} /$ frame, laser power $\leq 40 \mathrm{~mW}$ ). For axon imaging experiments with a tdTomato structural marker, we imaged both the green and red channel to visualize both the functional and structural markers, respectively.

For VNS pairing while imaging excitatory neurons, the speaker was $\sim 10 \mathrm{~cm}$ away from the ear contralateral to the window. A consistent region of excitatory neurons in layer 2/3 of $A 1$ (based on vasculature and relative orientation of neurons) was imaged over all days of pairing. For baseline imaging, pure tones (70 dB SPL, 4-64 kHz, $250 \mathrm{~ms}, 10 \mathrm{~ms}$ cosine on/off ramps, quarter-octave spacing, 10 trials for each frequency) were delivered 
in pseudo-random sequence every 20 frames. During pairing, one frequency (chosen based on the initial tuning of the area) was played concurrently with VNS every 10 frames for 5 minutes.

Excitatory neuron imaging data were first motion-corrected using the NoRMCorre algorithm ${ }^{78}$. Regions of interest (ROIs) were automatically detected using the CalmAn algorithm ${ }^{79}$ and manually verified. Additional ROls were manually drawn on an average image of all motion-corrected images. Calcium fluorescence was extracted from all ROIs. Semi-automated data analysis was performed using custom Matlab (MathWorks) software. For each ROI, we corrected for potential neuropil contamination as previously described ${ }^{80}$. The $\Delta F / F(\%)$ was calculated as the average change in fluorescence during the stimulus epoch relative to the $750 \mathrm{~ms}$ immediately prior to stimulus onset: $\Delta \mathrm{F} / \mathrm{F}$ $(\%)=\left(\left(F_{t}-F_{0}\right) / F_{0}\right) * 100$. ROls were included in additional analysis if they had a significant response (both $\mathrm{p}<0.05$ Student's two-tailed, paired t-test comparing activity during any stimulus and pre-stimulus epochs and had a mean $\Delta F / F$ equal to $5 \%$ or above for all trials with a particular frequency).

\section{Circuit tracing}

For monosynaptic pseudotype tracing studies, $0.5 \mu \mathrm{L}$ of a mix of pAAV-TREtight0mTagBFP2-B19G (diluted 1:20 in dPBS; Addgene: 100799-AAV1) and pAAV-syn-FLEXsplitTVA-EGFP-tTA (diluted 1:200 in dPBS; Addgene: 100798-AAV1) was injected into basal forebrain (AP: $-0.5 \mathrm{~mm}$, ML: $-1.8 \mathrm{~mm}$, DV: $-4.5 \mathrm{~mm}$ from brain surface) of ChATCre mice. After one week, $0.25 \mu \mathrm{L}$ of EnvA G-Deleted Rabies-mCherry (diluted 1:5 in dPBS; Addgene: 32636) was injected in the basal forebrain using the same coordinates. For mapping potential connectivity between basal forebrain, locus coeruleus and nucleus tractus solitarius, $0.75 \mu \mathrm{L}$ of rgAAV-FLEX-tdTomato (diluted 1:3 in dPBS; Addgene number: 28306) was injected in TH-Cre mice in basal forebrain (AP: $-0.5 \mathrm{~mm}$, ML: -1.8 $\mathrm{mm}$, DV: $-4.5 \mathrm{~mm}$ from brain surface) using either a Hamilton syringe $(5 \mu \mathrm{L})$ or Nanoject (Drummond Scientific; Part number: 3-000-207).

Animals were deeply anaesthetized with isoflurane and then transcardially perfused with phosphate buffered saline (1x PBS) followed by $4 \%$ paraformaldehyde (PFA) in PBS. Animals injected for circuit tracing studies were perfused 3-6 weeks after viral injection. Animals injected for optogenetics behavioral experiments were perfused after completion of behavioral training. After at least 12 hours in 4\% PFA, brains were either transferred to PBS for sectioning with a vibratome or to a $30 \%$ sucrose-PBS solution for 24-48 hours to prepare for cryosectioning. For cryosectioning, brains were embedded in Tissue-Plus ${ }^{\mathrm{TM}}$ O.C.T. Compound medium (Thermo Fisher Scientific, Item\# 23-730) and sectioned using a cryostat (Leica). All sections were cut at $50 \mu \mathrm{m}$.

For animals injected with monosynaptic pseudotyped rabies, brain sections were washed with PBS (3x10 min at room temperature) and incubated for 2 hours at room 
temperature in blocking solution containing 5\% normal goat serum (Millipore Sigma, Item \# G6767) in 1\% Triton X-100 (Millipore Sigma, Item \#11332481001) dissolved in PBS. Brain slices containing basal forebrain were incubated in primary antibody (1:500 dilution of $3 \%$ normal goat serum in 1\% Triton X100 dissolved in PBS of chicken anti-GFP IgY, Abcam catalog \# ab13970 and rabbit anti-mCherry IgG, Abcam catalog \# ab167453) for 24-48 hours at $4^{\circ} \mathrm{C}$. Afterwards, slices were washed and incubated for 1-2 hours at room temperature in secondary antibody (1:500 dilution in PBS, goat anti-chicken IgY Alexa Fluor 488, Thermo Fisher Scientific cat. \# A11039 and goat anti-Rabbit lgG $(\mathrm{H}+\mathrm{L})$ CrossAbsorbed Secondary Antibody, Alexa Fluor 555, Thermo Fisher Scientific Part number \# A21428). Brain slices containing locus coeruleus and NTS were incubated in primary antibody (1:500 dilution of 3\% normal goat serum in 1\% Triton X100 dissolved in PBS, rabbit anti-mCherry lgG, Abcam catalog \# ab167453) for $24-48$ hours at $4^{\circ} \mathrm{C}$. Slices were washed and incubated for 1-2 hours at room temperature in secondary antibody (1:500 dilution, goat anti-Rabbit IgG $(\mathrm{H}+\mathrm{L})$ Cross-Absorbed Secondary Antibody, Alexa Fluor 555, Thermo Fisher Scientific Part number \# A21428). Finally, slides were washed and coverslipped using VECTASHIELD Antifade Mounting Medium with DAPI (Vector Labs Part number\#: $\mathrm{H}-1200-10)$. For all other animals, brain sections were washed with PBS and mounted using VECTASHIELD Antifade Mounting Medium with DAPI. Slides were imaged using a Carl Zeiss LSM 700 confocal microscope with four solid-state lasers $(405 / 444,488,555,639 \mathrm{~nm})$ and appropriate filter sets.

\section{Optogenetic stimulation or inhibition of basal forebrain cholinergic neurons}

To stimulate auditory cortical projecting cholinergic neurons, $1.0 \mu \mathrm{L}$ of AAVrg.EF1a. doublefloxed.hChR2(H134R).EYFP.WPRE-HGHpA (diluted 1:2 with dPBS; Addgene number: 20298) was injected into auditory cortex as described above in ChAT-Cre animals. For optogenetic inhibition, $0.50 \mu \mathrm{L}$ of AAV5.FLEX.ArchT.tdTomato (diluted 1:2 with dPBS; Addgene number: 28305) was injected in basal forebrain of ChAT-Cre animals using the coordinates described above. In both cases, optic fibers were implanted $50 \mu \mathrm{m}$ above basal forebrain and the animal was head-posted. Optic fibers were custom made of glass fibers (200 $\mu \mathrm{m}$ core; Thorlabs FT200UMT) fitted with zirconia LC connectors (Precision Fiber Products MM-FER2007C-2300), secured using glue (Krazy Glue). All fibers used had at least an $80 \%$ efficiency prior to implantation.

For optogenetic stimulation during behavior, cholinergic basal forebrain neurons were bilaterally stimulated in the same way as VNS pairing (500 ms centered around the tone at $30 \mathrm{~Hz}$; two blocks of 50 stimulation trials surrounded by unstimulated trials). For optogenetic inactivation experiments, cholinergic basal forebrain neurons were bilaterally inhibited using a 500 ms pulse, presented in conjunction with VNS pairing. Light (either blue or yellow) was delivered using a driver (Thorlabs LEDD1B), fiber coupled LED (Thorlabs M470F3 or M565F3), a patch cable (Thorlabs M129L01), and a bifurcated fiber 
bundle (Thorlabs BFYL2LF01) connected to the fibers using a mating sleeve (Thorlabs ADAL1). Laser power was calibrated across days and between 1-3 $\mathrm{mW}$ for all optogenetics experiments during behavior.

\section{Author Contributions}

K.A.M., J.K.S and R.C.F. designed the experiments. K.A.M, E.S.P, and J.K.S. performed the experiments. K.A.M. performed analysis. E.S.P. and S.S.F. provided technical assistance. N.Z.T., E.S.P., D.A.M., and M.J.M. designed and validated the VNS cuff electrode. K.A.M and R.C.F. wrote the manuscript.

\section{Acknowledgments}

We thank A. Agha, C.L. Ebbesen, E. Glennon, E. Kay-Rivest, R.A. Kolaric, K.I. Nagel, D.H. Sanes, D.M. Schneider, M.A. Svirsky, J.T. Roland, and S. Valtcheva for comments, discussions, and/or technical assistance. We thank the GENIE Program, Janelia Research Campus, and the Howard Hughes Medical Institute for provision of GCaMP6s and Scanlmage. This work was funded by grants from the Defense Advanced Research Projects Agency (N66001-17-2-4010 to R.C.F., D.A.M., and M.J.M.), the National Institute on Deafness and Other Communication Disorders (DC012557 to R.C.F.), and the National Science Foundation (to K.A.M. and J.K.S.). 


\section{References}

1. Fu, Y.-X. et al. Temporal Specificity in the Cortical Plasticity of Visual Space Representation. Science 296, 1999-2003 (2002).

2. Gilbert, C. D., Li, W. \& Piech, V. Perceptual learning and adult cortical plasticity. The Journal of Physiology 587, 2743-2751 (2009).

3. Khan, A. G. et al. Distinct learning-induced changes in stimulus selectivity and interactions of GABAergic interneuron classes in visual cortex. Nature Neuroscience 21, 851-859 (2018).

4. Poort, J. et al. Learning Enhances Sensory and Multiple Non-sensory Representations in Primary Visual Cortex. Neuron 86, 1478-1490 (2015).

5. Schoups, A., Vogels, R., Qian, N. \& Orban, G. Practising orientation identification improves orientation coding in V1 neurons. Nature 412, 549-553 (2001).

6. Yan, Y. et al. Perceptual training continuously refines neuronal population codes in primary visual cortex. Nature Neuroscience 17, 1380-1387 (2014).

7. Yao, H. \& Dan, Y. Stimulus Timing-Dependent Plasticity in Cortical Processing of Orientation. Neuron 32, 315-323 (2001).

8. Recanzone, G. H., Schreiner, C. E. \& Merzenich, M. M. Plasticity in the frequency representation of primary auditory cortex following discrimination training in adult owl monkeys. The Journal of Neuroscience 13, 87 LP - 103 (1993).

9. Polley, D. B., Steinberg, E. E. \& Merzenich, M. M. Perceptual Learning Directs Auditory Cortical Map Reorganization through Top-Down Influences. The Journal of Neuroscience 26, 4970 LP - 4982 (2006).

10. Karmarkar, U. R. \& Buonomano, D. V. Temporal specificity of perceptual learning in an auditory discrimination task. Learning and Memory 10, 141-147 (2003).

11. Edeline, J.-M., Pham, P. \& Weinberger, N. M. Rapid development of learninginduced receptive field plasticity in the auditory cortex. Behavioral Neuroscience vol. 107 539-551 (1993).

12. Bakin, J. S. \& Weinberger, N. M. Classical conditioning induces CS-specific receptive field plasticity in the auditory cortex of the guinea pig. Brain Research 536, 271-286 (1990).

13. Recanzone, G. H., Merzenich, M. M., Jenkins, W. M., Grajski, K. A. \& Dinse, H. R. Topographic reorganization of the hand representation in cortical area $3 \mathrm{~b}$ owl monkeys trained in a frequency-discrimination task. Journal of Neurophysiology 67, 1031-1056 (1992).

14. Feldman, D. E. \& Brecht, M. Map Plasticity in Somatosensory Cortex. Science 310, 810-815 (2005).

15. Wilson, D. A. \& Stevenson, R. J. Olfactory perceptual learning: the critical role of memory in odor discrimination. Neuroscience \& Biobehavioral Reviews 27, 307328 (2003). 
16. Karni, A. \& Sagi, D. The time course of learning a visual skill. Nature 365, 250252 (1993).

17. van Kerkoerle, T., Marik, S. A., Meyer zum Alten Borgloh, S. \& Gilbert, C. D. Axonal plasticity associated with perceptual learning in adult macaque primary visual cortex. Proceedings of the National Academy of Sciences 115, 10464 LP 10469 (2018).

18. Tsodyks, M. \& Gilbert, C. Neural networks and perceptual learning. Nature 431, 775-781 (2004).

19. Bao, S., Chang, E. F., Woods, J. \& Merzenich, M. M. Temporal plasticity in the primary auditory cortex induced by operant perceptual learning. Nature Neuroscience 7, 974-981 (2004).

20. David, S. V., Fritz, J. B. \& Shamma, S. A. Task reward structure shapes rapid receptive field plasticity in auditory cortex. Proceedings of the National Academy of Sciences 109, 2144 LP - 2149 (2012).

21. Froemke, R. C. Plasticity of Cortical Excitatory-Inhibitory Balance. Annual Review of Neuroscience 38, 195-219 (2015).

22. Magee, J. C. \& Grienberger, C. Synaptic Plasticity Forms and Functions. Annual Review of Neuroscience 43, 95-117 (2020).

23. Kilgard, M. P. \& Merzenich, M. M. Cortical Map Reorganization Enabled by Nucleus Basalis Activity. Science 279, 1714-1718 (1998).

24. Reed, A. et al. Cortical Map Plasticity Improves Learning but Is Not Necessary for Improved Performance. Neuron 70, 121-131 (2011).

25. Edeline, J.-M., Hars, B., Maho, C. \& Hennevin, E. Transient and prolonged facilitation of tone-evoked responses induced by basal forebrain stimulations in the rat auditory cortex. Experimental Brain Research 97, 373-386 (1994).

26. Bakin, J. S. \& Weinberger, N. M. Induction of a physiological memory in the cerebral cortex by stimulation of the nucleus basalis. Proceedings of the National Academy of Sciences 93, 11219 LP - 11224 (1996).

27. Goard, M. \& Dan, Y. Basal forebrain activation enhances cortical coding of natural scenes. Nature Neuroscience 12, 1444-1449 (2009).

28. Pinto, L. et al. Fast modulation of visual perception by basal forebrain cholinergic neurons. Nature Neuroscience 16, 1857-1863 (2013).

29. Guo, W., Robert, B. \& Polley, D. B. The Cholinergic Basal Forebrain Links Auditory Stimuli with Delayed Reinforcement to Support Learning. Neuron 103, 1164-1177.e6 (2019).

30. Robert, B. et al. A functional topography within the cholinergic basal forebrain for encoding sensory cues and behavioral reinforcement outcomes. eLife 10, e69514 (2021). 
31. Kuchibhotla, K. V. et al. Parallel processing by cortical inhibition enables contextdependent behavior. Nature Neuroscience 20, 62-71 (2017).

32. Letzkus, J. J. et al. A disinhibitory microcircuit for associative fear learning in the auditory cortex. Nature 480, 331-335 (2011).

33. Poorthuis, R. B., Enke, L. \& Letzkus, J. J. Cholinergic circuit modulation through differential recruitment of neocortical interneuron types during behaviour. The Journal of Physiology 592, 4155-4164 (2014).

34. Thiel, C. M., Friston, K. J. \& Dolan, R. J. Cholinergic Modulation of ExperienceDependent Plasticity in Human Auditory Cortex. Neuron 35, 567-574 (2002).

35. Froemke, R. C. et al. Long-term modification of cortical synapses improves sensory perception. Nature Neuroscience 16, 79-88 (2013).

36. Wilson, D. A., Fletcher, M. L. \& Sullivan, R. M. Acetylcholine and Olfactory Perceptual Learnin. Learning and Memory 11, 28-34 (2004).

37. Penry, J. K. \& Dean, J. C. Prevention of Intractable Partial Seizures by Intermittent Vagal Stimulation in Humans: Preliminary Results. Epilepsia 31, S40S43 (1990).

38. Fisher, B., DesMarteau, J. A., Koontz, E. H., Wilks, S. J. \& Melamed, S. E. Responsive Vagus Nerve Stimulation for Drug Resistant Epilepsy: A Review of New Features and Practical Guidance for Advanced Practice Providers. Frontiers in Neurology 11, (2021).

39. O'Reardon, J. P., Cristancho, P. \& Peshek, A. D. Vagus Nerve Stimulation (VNS) and Treatment of Depression: To the Brainstem and Beyond. Psychiatry (Edgmont (Pa. : Township)) 3, 54-63 (2006).

40. Meyers, E. C. et al. Vagus Nerve Stimulation Enhances Stable Plasticity and Generalization of Stroke Recovery. Stroke 49, 710-717 (2018).

41. Engineer, N. D. et al. Reversing pathological neural activity using targeted plasticity. Nature 470, 101-104 (2011).

42. Nichols, J. A. et al. Vagus nerve stimulation modulates cortical synchrony and excitability through the activation of muscarinic receptors. Neuroscience 189, 207-214 (2011).

43. Lai, J. \& David, S. V. Short-Term Effects of Vagus Nerve Stimulation on Learning and Evoked Activity in Auditory Cortex. eneuro 8, ENEURO.0522-20.2021 (2021).

44. Lockard, J. S., Congdon, W. C. \& DuCharme, L. L. Feasibility and Safety of Vagal Stimulation In Monkey Model. Epilepsia 31, S20-S26 (1990).

45. Mughrabi, I. T. et al. Development and characterization of a chronic implant mouse model for vagus nerve stimulation. eLife 10, e61270 (2021).

46. Bowles, S. et al. Vagus nerve stimulation accelerates motor learning through cholinergic modulation. bioRxiv 2021.10.28.466306 (2021)

doi:10.1101/2021.10.28.466306. 
47. Mridha, Z. et al. Graded recruitment of pupil-linked neuromodulation by parametric stimulation of the vagus nerve. Nature Communications 12, 1539 (2021).

48. Collins, L., Boddington, L., Steffan, P. J. \& McCormick, D. Vagus nerve stimulation induces widespread cortical and behavioral activation. Current Biology 31, 2088-2098.e3 (2021).

49. Buell, E. P. et al. Vagus Nerve Stimulation Rate and Duration Determine whether Sensory Pairing Produces Neural Plasticity. Neuroscience 406, 290-299 (2019).

50. Hays, S. A. et al. Vagus nerve stimulation during rehabilitative training enhances recovery of forelimb function after ischemic stroke in aged rats. Neurobiology of Aging 43, 111-118 (2016).

51. Hays, S. A. et al. Vagus nerve stimulation during rehabilitative training improves functional recovery after intracerebral hemorrhage. Stroke 45, 3097-3100 (2014).

52. Groves, D. A., Bowman, E. M. \& Brown, V. J. Recordings from the rat locus coeruleus during acute vagal nerve stimulation in the anaesthetised rat. Neuroscience Letters 379, 174-179 (2005).

53. Hulsey, D. R. et al. Parametric characterization of neural activity in the locus coeruleus in response to vagus nerve stimulation. Experimental Neurology 289, 21-30 (2017).

54. van Bockstaele, E. J., Peoples, J. \& Telegan, P. Efferent projections of the nucleus of the solitary tract to peri-Locus coeruleus dendrites in rat brain: Evidence for a monosynaptic pathway. Journal of Comparative Neurology 412 , 410-428 (1999).

55. Schwarz, L. A. \& Luo, L. Organization of the Locus Coeruleus-Norepinephrine System. Current Biology 25, R1051-R1056 (2015).

56. King, J. et al. Rodent auditory perception: Critical band limitations and plasticity. Neuroscience 296, 55-65 (2015).

57. Heffner, H. \& Masterton, B. Hearing in Glires: Domestic rabbit, cotton rat, feral house mouse, and kangaroo rat. The Journal of the Acoustical Society of America 68, 1584-1599 (1980).

58. Klein, A. S., Dolensek, N., Weiand, C. \& Gogolla, N. Fear balance is maintained by bodily feedback to the insular cortex in mice. Science 374, 1010-1015 (2021).

59. Martins, A. R. O. \& Froemke, R. C. Coordinated forms of noradrenergic plasticity in the locus coeruleus and primary auditory cortex. Nature Neuroscience 18, 1483-1492 (2015).

60. Yakunina, N., Kim, S. S. \& Nam, E.-C. Optimization of Transcutaneous Vagus Nerve Stimulation Using Functional MRI. Neuromodulation: Technology at the Neural Interface 20, 290-300 (2017). 
61. Hulsey, D. R. et al. Reorganization of Motor Cortex by Vagus Nerve Stimulation Requires Cholinergic Innervation. Brain Stimul 9, 174-181 (2016).

62. Do, J. P. et al. Cell type-specific long-range connections of basal forebrain circuit. eLife 5, e13214 (2016).

63. Kim, J.-H. et al. Selectivity of Neuromodulatory Projections from the Basal Forebrain and Locus Ceruleus to Primary Sensory Cortices. The Journal of Neuroscience 36, 5314 LP - 5327 (2016).

64. Gielow, M. R. \& Zaborszky, L. The Input-Output Relationship of the Cholinergic Basal Forebrain. Cell Reports 18, 1817-1830 (2017).

65. Laszlovszky, T. et al. Distinct synchronization, cortical coupling and behavioral function of two basal forebrain cholinergic neuron types. Nature Neuroscience 23, 992-1003 (2020).

66. Wickersham, I. R., Finke, S., Conzelmann, K.-K. \& Callaway, E. M. Retrograde neuronal tracing with a deletion-mutant rabies virus. Nature Methods 4, 47-49 (2007).

67. Liu, K. et al. Lhx6-positive GABA-releasing neurons of the zona incerta promote sleep. Nature 548, 582-587 (2017).

68. Hecht, S., Shlaer, S. \& Pirenne, M. H. Energy, Quanta and Vision. Journal of General Physiology 25, 819-840 (1942).

69. Baylor, D. A., Lamb, T. D. \& Yau, K. W. Responses of retinal rods to single photons. The Journal of Physiology 288, 613-634 (1979).

70. Reimer, J. et al. Pupil fluctuations track rapid changes in adrenergic and cholinergic activity in cortex. Nature Communications 7, 13289 (2016).

71. McGinley, M. J. et al. Waking State: Rapid Variations Modulate Neural and Behavioral Responses. Neuron 87, 1143-1161 (2015).

72. Prechtl, J. C. \& Powley, T. L. The fiber composition of the abdominal vagus of the rat. Anatomy and Embryology 181, 101-115 (1990).

73. Cunningham, J. T., Mifflin, S. W., Gould, G. G. \& Frazer, A. Induction of c-Fos and $\triangle$ FosB Immunoreactivity in Rat Brain by Vagal Nerve Stimulation.

Neuropsychopharmacology 33, 1884-1895 (2008).

74. Settell, M. L. et al. Functional vagotopy in the cervical vagus nerve of the domestic pig: Implications for the study of vagus nerve stimulation. Journal of Neural Engineering 17, (2020).

75. Chang, R. B., Strochlic, D. E., Williams, E. K., Umans, B. D. \& Liberles, S. D. Vagal Sensory Neuron Subtypes that Differentially Control Breathing. Cell 161, 622-633 (2015).

76. Carter, M. E. et al. Tuning arousal with optogenetic modulation of locus coeruleus neurons. Nature Neuroscience 13, 1526-1533 (2010). 
77. Yap, J. Y. Y. et al. Critical Review of Transcutaneous Vagus Nerve Stimulation: Challenges for Translation to Clinical Practice. Frontiers in Neuroscience vol. 14 (2020).

78. Pnevmatikakis, E. A. \& Giovannucci, A. NoRMCorre: An online algorithm for piecewise rigid motion correction of calcium imaging data. Journal of Neuroscience Methods 291, 83-94 (2017).

79. Giovannucci, A. et al. CalmAn an open source tool for scalable calcium imaging data analysis. eLife 8, e38173 (2019).

80. Kerlin, A. M., Andermann, M. L., Berezovskii, V. K. \& Reid, R. C. Broadly Tuned Response Properties of Diverse Inhibitory Neuron Subtypes in Mouse Visual Cortex. Neuron 67, 858-871 (2010). 